\title{
Stereochemical Control of the Passerini Reaction
}

\author{
Peter R. Andreana, Chang C. Liu, Stuart L. Schreiber*
}

Howard Hughes Medical Institute, Center for Chemical Methodology and Library Development (CMLD-HU) \& Broad Institute of Harvard and MIT, Department of Chemistry and Chemical Biology, Harvard University, Cambridge, MA.

\section{Supporting Information}

Materials and Methods. All reagents were purchased from either Aldrich or Strem Chemicals. Amino indanol based pybox ligand (4) was synthesized according to the procedure by Davies et al. (as described in J. Org. Chem. 1996, 61, 9629-9630). All solvents used were obtained from the Solvent Dispensing System (SDS). Methylene chloride was passed through two packed columns of neutral alumina (as described in Organometallics 1996, 15, 1518-1520). Except as otherwise indicated, reactions were carried out under argon. Molecular Sieves, AW-300 $1.6 \mathrm{~mm}$ pellets $(1 \mathrm{~g})$ were dried using the microwave for 3 min and then flame dried under vacuum for a total of $3 \mathrm{~h}$.

All reactions were monitored by thin layer chromatography using $0.25 \mathrm{~mm}$ E. Merck precoated silica gel 60 (particle size $0.040-0.063 \mathrm{~mm}$ ). Column chromatography was performed using Merck $60 \AA$ (230-400 mesh ASTM) silica gel or an Isco CombiFlash® Companion system. RediSep disposable Flash columns or Biotage FLASH+TM HPFCTM Cartridges were used interchangeably for purification purposes. Yields refer to chromatographically and spectroscopically pure compounds, except as noted. Concentration refers to the removal of solvent using a Büchi rotary evaporator followed by use of a vacuum pump at approximately 1 torr. 
Proton and carbon-13 NMR spectra were recorded on Varian Mercury 400, Varian Mercury 500 and Varian Unity/Inova 600 spectrometers. Proton and carbon-13 chemical shifts are reported in $\delta$ values relative to chloroform (7.26 ppm). 1D and 2D NMR experiments were configured using the GLIDE NMR software package VNMR 6.1B Software provided by Varian. Optical rotations were measured using a $2 \mathrm{~mL}$ cell with a $1 \mathrm{dm}$ path length on a JASCO DIP 370 digital polarimeter. High resolution mass spectra were obtained with JEOL AX-505 and JEOL SX-102 spectrometers. IR data was collected on a Nicolet 5PC FT-IR Spectrometer using EZ OMNIC software version 4.1b. All samples were dissolved in methylenechloride and run neat.

X-Ray Crystallographic Data. All the crystal structure information for (+/-)-18 and (tert-butylcarbamoyl)(5-bromothiophen-2-yl)methyl benzoate will be submitted to the Cambridge Crystal Structure Data Base following the instructions given by the Journal of the American Chemical Society (J. Am. Chem. Soc. 1999, 121, 7A-12A).

General Procedure I. Preparation of $\alpha$-acyloxycarboxamides (Rac). In a $25 \mathrm{~mL}$ flame dried r.b.f. equipped with a stir bar, aldehyde (0.52 mmol) was dissolved in $2.5 \mathrm{~mL}$ of methylenechloride. To this solution were added, acid (0.52 mmol), and isocyanide (0.45 mmol). The reaction was stirred for 24 to $48 \mathrm{~h}$. The reaction was monitored for complete consumption of the isocyanide, based on TLC analysis using a 1:1, ethyl acetate:hexanes eluent. Sodium hypochlorite $(\mathrm{NaOCl})$ was used to denature the isocyanide or similarly a $1 \mathrm{M} \mathrm{HCl}$ solution was used. The reaction mix was diluted with water and methylenechloride and extracted. After removal of solvent under reduced 
pressure, the crude reaction mixture was applied directly to a silica gel column. The major product (as denoted by the TLC) was separated by column chromatography (silica gel, 9:1 - 1:3 hexanes:ethyl acetate).

General Procedure II. Preparation of $\alpha$-acyloxycarboxamides (Enan). A $25 \mathrm{~mL}$ flame dried r.b.f. under argon and equipped with a stir bar was charged with $\cong 7.0-9.0$ mL methylenechloride, $\mathrm{Cu}(\mathrm{OTf})_{2}(20 \mathrm{~mol} \%)$ and indane (pybox) ligand 4 (20 mol \%). ${ }^{1}$ The mixture was stirred until the components were completely dissolved (heating the solution to $38{ }^{\circ} \mathrm{C}$ was necessary). Aldehyde (0.40-0.50 mmol) was dissolved and the solution was cooled to $0{ }^{\circ} \mathrm{C}$ and allowed to stand for 30 min at which time AW-300 MS (molecular sieves $\cong 400 \mathrm{mg}$ ) were added. In a separate, dry r.b.f. (under argon), the acid (0.40-0.50 mmol) and isocyanide $(0.40-0.50 \mathrm{mmol}, 0.95$ equiv) were dissolved in $\cong 3.0$ mL of methylenechloride. AW-300 MS (100 mg) were added and the mixture was either shaken or stirred for $30 \mathrm{~min}$. This mixture was drawn into a gas syringe and delivered to the first solution via a syringe pump at a rate that ended after $4 \mathrm{~h}$. The reaction was stirred for 8 to 18 h during which time TLC analysis, using a 1:1, ethyl acetate:hexanes eluent, was conducted. When the TLC denoted completion, $2.0 \mathrm{~mL}$ of sodium hypochlorite $(\mathrm{NaOCl})$ was added and the mixture was allowed to stir for a further $1 \mathrm{~h}$. After removal of solvent under reduced pressure, the crude reaction mixture was applied directly to a silica gel column. The major product (as denoted by the TLC) was separated by gradient flash column chromatography (silica gel, 9:1 - 1:3 hexanes:ethyl acetate).

\footnotetext{
1 Ref 10 in manuscript.
} 


\section{Synthesis of 1-(4-methoxyphenylcarbamoyl)-2-(benzyloxy)ethyl benzoate (8)}<smiles>COc1ccc(NC(=O)C(COc2ccccc2)OC(=O)c2ccccc2)cc1</smiles>

8 Following General Procedure II, a mixture of isocyanide 7 (60 mg, 0.45 mmol, 1.0 equiv), benzoic acid (5) (58 mg, 0.47 mmol, 1.05 equiv) aldehyde 6 (71 mg, $66 \mu \mathrm{L}, 0.47$ mmol, 1.05 equiv) and $20 \mathrm{~mol} \%$ of catalyst 4 in $\mathrm{CH}_{2} \mathrm{Cl}_{2}$

was stirred for 18 hrs. After removal of solvent followed by silica gel chromatography, 8 (170 mg, 93\%) $)^{2}$ was obtained as a white solid.

\section{Data for 8:}

${ }^{1}$ H NMR: $\quad\left(500 \mathrm{MHz}, \mathrm{CDCl}_{3}\right)$

$\delta$ 8.14-8.12 (dd, $J=1.0,8.5 \mathrm{~Hz}, 2 \mathrm{H}$, aryl); 7.92 (bs, 1H, NH); 7.66-7.63 (m, 2H, aryl); 7.52-7.47 (m, 2H, aryl); 7.43-7.40 (m, 2H, aryl); 7.31-7.26 (m, 4H, aryl); 6.86-6.85 (d, $J=8.5 \mathrm{~Hz}, 2 \mathrm{H}$, aryl); 5.70 (t, $J=4.0,1 \mathrm{H}, \mathrm{CH}$ ); 4.62 (s, $2 \mathrm{H}, \mathrm{CH}_{2}$ ); 4.07 (dd, $J=4.5,6.5 \mathrm{~Hz}, 1 \mathrm{H}, \mathrm{CH}_{2}$ ); 3.99 (dd, $J=4.5, J$ $\left.=6.5,1 \mathrm{H}, \mathrm{CH}_{2}\right) ; 3.79\left(\mathrm{~s}, 3 \mathrm{H}, \mathrm{CH}_{3}\right)$.

${ }^{13}$ C NMR: $\quad\left(100 \mathrm{MHz}, \mathrm{CDCl}_{3}\right)$ $\delta$ 171.3, 163.9, 156.9, 133.9, 131.9, 130.4, 130.2, 129.6, 129.4, 129.0, 128.9, 128.7, 127.9, 127.2, 122.3, 114.5, 73.8, 69.5, 55.7.

IR: (neat)

$v_{\max }=1726,1690,1512,1246 \mathrm{~cm}^{-1}$

TLC: $\quad \mathrm{R} f=0.30$ (silica gel, EtOAc:Hex 1:2)

HRMS: $\quad$ EIMS calculated for $\mathrm{C}_{24} \mathrm{H}_{23} \mathrm{NO}_{5}{ }^{+}[\mathrm{M}+\mathrm{H}]^{+}:$406.1655, found: 406.1653

HPLC: $\quad(+/-)-8 t_{\mathrm{R}} 27.41 \min (48.7 \%) ; t_{\mathrm{R}} 30.72 \min (51.3 \%)$

\footnotetext{
${ }^{2}$ Based on entry 7 of Table 1 in manuscript.
} 
(+)-8 $t_{\mathrm{R}} 28.72 \min (98.4 \%) ; t_{\mathrm{R}} 32.23 \min (1.6 \%)$ (Daicel Chiralcel OD 0.46 $\mathrm{cm} \phi$ x 25 cm, 5\% IPA in Hexane, $1 \mathrm{~mL} / \mathrm{min}$; isocratic)

OR: $\quad[\alpha]_{\mathrm{D}}^{22}=+17.7^{\mathrm{o}}\left(\mathrm{CHCl}_{3}, \mathrm{c}=2.21\right)$
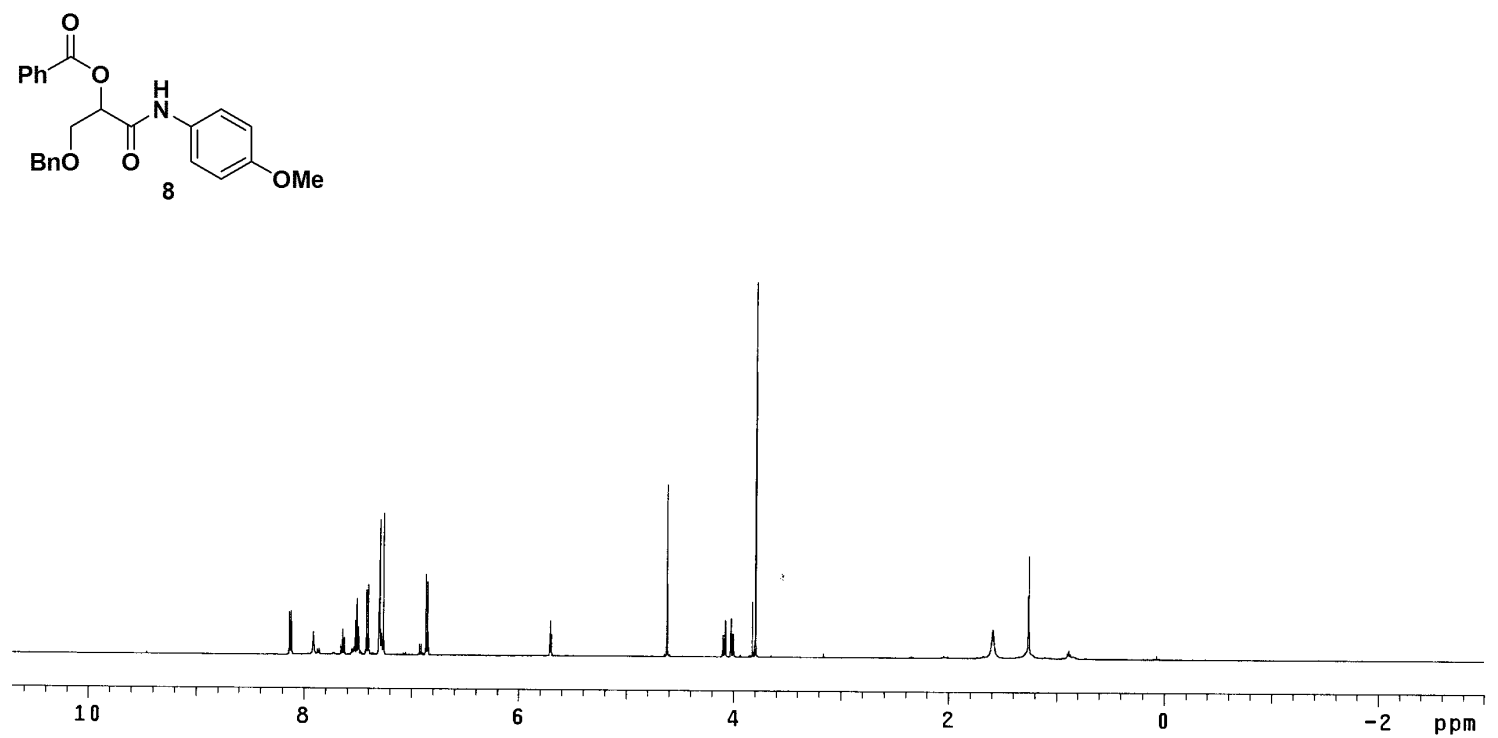

\section{Synthesis of (benzylcarbamoyl)(furan-2-yl)methyl 2-phenylacetate (16)}<smiles>O=C(Cc1ccccc1)OC(C(=O)NCc1ccccc1)c1ccco1</smiles>

16

Following General Procedure II, a mixture of isocyanide 11 (58 mg, $56 \mu \mathrm{L}, 0.50$ mmol, 1.0 equiv), phenyl acetic acid (9) (71 mg, 0.525 mmol, 1.05 equiv), aldehyde 10 (50 mg, $58 \mu \mathrm{L}, 0.525$

mmol, 1.05 equiv) and $20 \mathrm{~mol} \%$ of catalyst 4 in $\mathrm{CH}_{2} \mathrm{Cl}_{2}$ was

stirred for 21 hrs. After removal of solvent followed by silica gel chromatography, 16 (145 mg, 83\%) was obtained as a white solid.

\section{Data for 16:}

${ }^{1} \mathrm{H}$ NMR: $\quad\left(400 \mathrm{MHz}, \mathrm{CDCl}_{3}\right)$

$\delta$ 7.42-7.35 (dd, $J=0.8,2.0 \mathrm{~Hz}, 2 \mathrm{H}$, aryl); 7.36-7.28 (m, 6H, aryl); 7.26 (s, 1H, furyl); 7.18-7.16 (m, 2H, aryl); 6.49 (d, $J=3.2 \mathrm{~Hz}, 1 \mathrm{H}$, furyl); 6.37- 
6.36 (dd, $J=2.0,3.2 \mathrm{~Hz}, 1 \mathrm{H}$, furyl); 6.27 (s, 1H, CH); 6.19 (bt, 1H, NH);

4.44-4.39 (dd, $\left.J=6.0,14.8 \mathrm{~Hz}, 1 \mathrm{H}, \mathrm{CH}_{2}-\mathrm{NH}\right)$; 4.35-4.30 (dd, $J=6.0,14.8$ $\left.\mathrm{Hz}, 1 \mathrm{H}, \mathrm{CH}_{2}-\mathrm{NH}\right) ; 3.70$ (s, 2H, $\mathrm{CH}_{2}$ ).

${ }^{13} \underline{\mathrm{C} \mathrm{NMR}}: \quad\left(100 \mathrm{MHz}, \mathrm{CDCl}_{3}\right)$

$\delta$ 169.6, 166.1, 148.0, 143.8, 137.6, 133.4, 129.3, 129.0, 127.9, 127.7, 111.8, 110.9, 69.0, 43.6, 41.5.

TLC: $\quad \mathrm{R} f \quad 0.37$ (Ethyl Acetate:Hexanes, 1:2)

LRMS: $\quad$ LC/EIMS calculated for $\mathrm{C}_{21} \mathrm{H}_{19} \mathrm{NO}_{4}{ }^{+}[\mathrm{M}+\mathrm{H}]^{+}: 350.1$, found: 349.7

HPLC: $\quad(+/-)-16 t_{\mathrm{R}} 13.78 \min (50.7 \%) ; t_{\mathrm{R}} 21.42 \min (49.3 \%)$

(-)-16 $t_{\mathrm{R}} 14.95 \min (18.8 \%) ; t_{\mathrm{R}} 22.13 \min (81.2 \%)$ (Daicel Chiralpak AS $0.46 \mathrm{~cm} \phi$ x $25 \mathrm{~cm}, 5 \%$ IPA in Hexane, $1 \mathrm{~mL} / \mathrm{min}$; isocratic)

OR: $\quad[\alpha]_{\mathrm{D}}^{22}=-12.2^{\circ}\left(\mathrm{CHCl}_{3}, \mathrm{c}=1.02\right)$
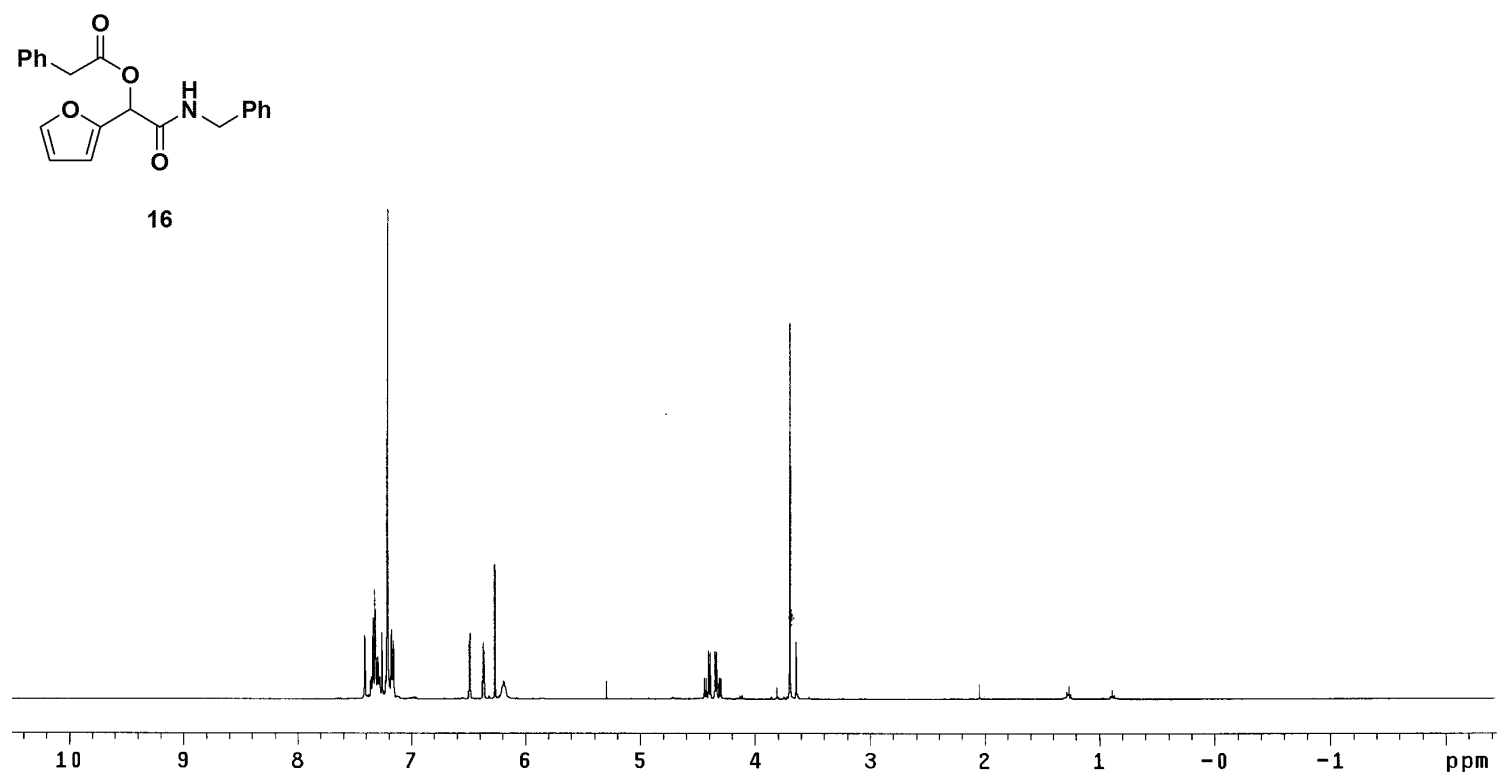


\section{Synthesis of 1-(benzylcarbamoyl)-2-(benzyloxy)ethyl 2-phenylacetate (17)}<smiles>O=C(Cc1ccccc1)OC(COc1ccccc1)C(=O)NCc1ccccc1</smiles>

17

Following General Procedure II, a mixture of isocyanide 11 (50 mg, 0.43 mmol, 1.0 equiv), phenyl acetic acid (9) (61 mg, 0.45 mmol, 1.05 equiv), aldehyde 6 (67.5 mg, $72 \mu \mathrm{L}, 0.45 \mathrm{mmol}$, 1.05 equiv) and 20 mol \% of catalyst 4 in $\mathrm{CH}_{2} \mathrm{Cl}_{2}$ was stirred for

24 hrs. After removal of solvent followed by silica gel chromatography, 17 (150 mg, 87\%) was obtained as a white solid.

\section{Data for 17:}

${ }^{1}$ H NMR: $\quad\left(400 \mathrm{MHz}, \mathrm{CDCl}_{3}\right)$ $\delta$ 7.37-7.20 (m, 11H, aryl); 7.15-7.13 (m, 4H, aryl); 6.18 (bt, 1H, NH); 5.43 (dd, $J=1.6,2.8 \mathrm{~Hz}, 1 \mathrm{H}, \mathrm{CH}$ ); 4.52 (s, 2H, $\mathrm{CH}_{2}$ ); 4.45-4.39 (dd, $J=$ 6.4, 14.4 Hz, 1H, CH); 4.28-4.22 (dd, $J=5.6,15.2 \mathrm{~Hz}, 1 \mathrm{H}, \mathrm{CH}$ ); 3.97-3.93 (dd, $J=4.4,10.4 \mathrm{~Hz}, 1 \mathrm{H}, \mathrm{CH}) ; 3.85-3.81$ (dd, $J=2.8,10.4,1 \mathrm{H}, \mathrm{CH}) ; 3.71$ (s, $2 \mathrm{H}, \mathrm{CH}_{2}$ ).

${ }^{13}$ C NMR: $\quad\left(100 \mathrm{MHz}, \mathrm{CDCl}_{3}\right)$ $\delta$ 170.0, 167.7, 137.9, 137.7, 133.6, 129.3, 129.1, 128.9, 128.7, 128.0, 127.9, 127.8, 127.7, 73.6, 73.6, 69.6, 43.4, 41.7.

TLC: $\quad \mathrm{Rf}=0.41$ (Ethyl Acetate:Hexanes, 1:2)

LRMS: $\quad$ LC/EIMS calculated for $\mathrm{C}_{25} \mathrm{H}_{25} \mathrm{NO}_{4}{ }^{+}[\mathrm{M}+\mathrm{H}]^{+}:$404.2, found: 403.7

HPLC: $\quad(+/-)-17 t_{\mathrm{R}} 26.60 \min (49.0 \%) ; t_{\mathrm{R}} 32.21 \min (51.0 \%)$

(+)-17 $t_{\mathrm{R}} 26.02 \min (85.8 \%) ; t_{\mathrm{R}} 31.13$ min (14.2\%) (Daicel Chiralpak ASH $0.46 \mathrm{~cm} \phi$ x 25 cm, 20\% IPA in Hexane, $1 \mathrm{~mL} / \mathrm{min}$; isocratic) 
OR: $\quad[\alpha]_{\mathrm{D}}^{22}=+23.3^{\circ}\left(\mathrm{CHCl}_{3}, \mathrm{c}=5.44\right)$
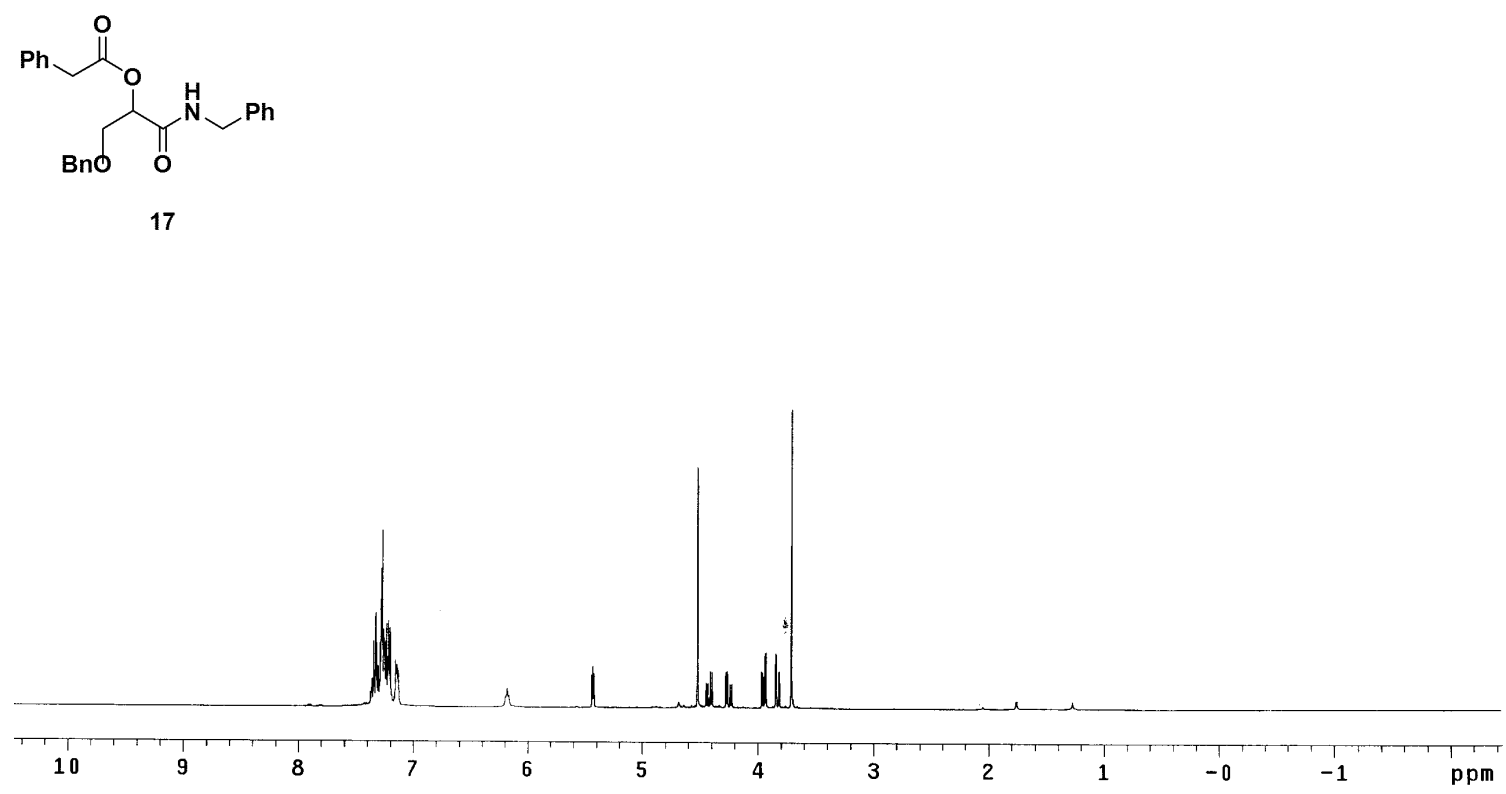

\section{Synthesis of (tert-butylcarbamoyl)(thiophen-2-yl)methyl benzoate (18) (x-ray)}

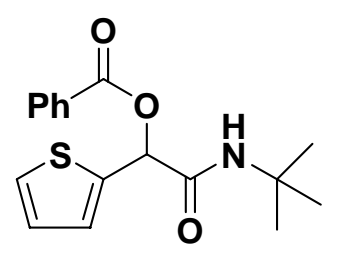

18 x-ray

Following General Procedure II, a mixture of isocyanide 13 (45 mg, $61 \mu \mathrm{L}, 0.54 \mathrm{mmol}, 1.0$ equiv), benzoic acid (5) (69 mg, $0.57 \mathrm{mmol}$, 1.05 equiv), aldehyde 12 (64 mg, $77 \mu \mathrm{L}, 0.57 \mathrm{mmol}, 1.05$ equiv) and $20 \mathrm{~mol} \%$ of catalyst 4 in $\mathrm{CH}_{2} \mathrm{Cl}_{2}$ was stirred for 24 hrs. After removal of solvent followed by silica gel chromatography, 18 (162 mg, 95\%) was obtained as a white solid.

$\underline{\text { Data for 18: }}$

${ }^{1} \mathrm{H}$ NMR: $\quad\left(400 \mathrm{MHz}, \mathrm{CDCl}_{3}\right)$

$\delta$ 8.13-8.07 (m, 2H, aryl); 7.64-7.59 (m, 1H, aryl); 7.50-7.46 (dt, $J=2.0$, 8.0 Hz, 2H, aryl); 7.33 (dd, $J=1.2,5.2 \mathrm{~Hz}, 1 \mathrm{H}$, aryl); 7.25-7.23 (m, $1 \mathrm{H}$, aryl); 7.00 (dd, $J=3.6,5.2 \mathrm{~Hz}, 1 \mathrm{H}$, aryl); 6.49 (s, 1H, CH); 6.01 (bs, $1 \mathrm{H}$, $\mathrm{NH}) ; 1.38\left(\mathrm{~s}, 9 \mathrm{H}, \mathrm{CH}_{3}\right)$ 
${ }^{13} \underline{\mathrm{C} \mathrm{NMR}}: \quad\left(100 \mathrm{MHz}, \mathrm{CDCl}_{3}\right)$

$\delta$ 166.7, 165.1, 138.0, 134.0, 130.4, 130.1, 128.9, 128.7, 128.0, 127.1, $72.0,52.0,28.9$

IR: $\quad$ (neat)

$v_{\max }=2968,2925,1723,1690,1452,1264,709 \mathrm{~cm}^{-1}$

TLC: $\quad \mathrm{R} f=0.45$ (Ethyl Acetate:Hexanes, $1: 2$ )

HRMS: $\quad$ EIMS calculated for $\mathrm{C}_{17} \mathrm{H}_{19} \mathrm{NO}_{3} \mathrm{~S}^{+}[\mathrm{M}+\mathrm{H}]^{+}:$317.1086, found: 317.1080

HPLC: $\quad(+/-)-18 t_{\mathrm{R}} 22.32 \min (49.0 \%) ; t_{\mathrm{R}} 24.42 \min (51.0 \%)$

(-)-18 $t_{\mathrm{R}} 24.67 \min (8.9 \%) ; t_{\mathrm{R}} 28.22 \min$ (91.1\%) (Diacel Chiralpak AS-H $0.46 \mathrm{~cm} \phi \times 25 \mathrm{~cm}, 2 \%$ IPA in Hexane, $0.7 \mathrm{~mL} / \mathrm{min}$; isocratic)

OR: $\quad[\alpha]_{\mathrm{D}}^{22}=-3.2^{\circ}\left(\mathrm{CHCl}_{3}, \mathrm{c}=1.13\right)$

$\underline{\text { X-Ray }} \quad$ Crystallographic data: monoclinic single crystal $(0.14$ x 0.10 x $0.10 \mathrm{~mm}$, Data: Space group P21/c, Unit cell constants $a=15.306(3), b=1.1614(18), c=$ 9.8395(16) $\AA, \alpha=90^{\circ}, \beta=94.631(3)^{\circ}, \gamma=90^{\circ}, V=1675.5(5) \AA^{3}, Z=4$, $\left.\mathrm{Dx}=1.258 \mathrm{~g} / \mathrm{cm}^{3}\right)$. X-ray data were collected using a Bruker SMART CCD (charge coupled device) based diffractometer equipped with an LT-3 low-temperature apparatus operating at $213 \mathrm{~K}$. A suitable crystal was hosen and mounted on a glass fiber using grease. Data were measured using mega scans of $0.3^{\circ}$ per frame for 30 seconds, such that a hemisphere was collected. A total of 1271 frames were collected with a maximum resolution of $0.76 \AA$. The first 50 frames were recollected at the end of data collection to monitor for decay. Cell parameters were retrieved using 
SMART software and refined using SAINT on all observed reflections. Data reduction was performed using the SAINT software which corrects for Lp and decay. The structures are solved by the direct method using the SHELXS-97 program and refined by least squares method on F2, SHELXL-97, incorporated in SHELXTL V6.10. The final agreement factors are $\mathrm{R}(\mathrm{F})=0.0730, \mathrm{Rw}(\mathrm{F})=0.1967$.
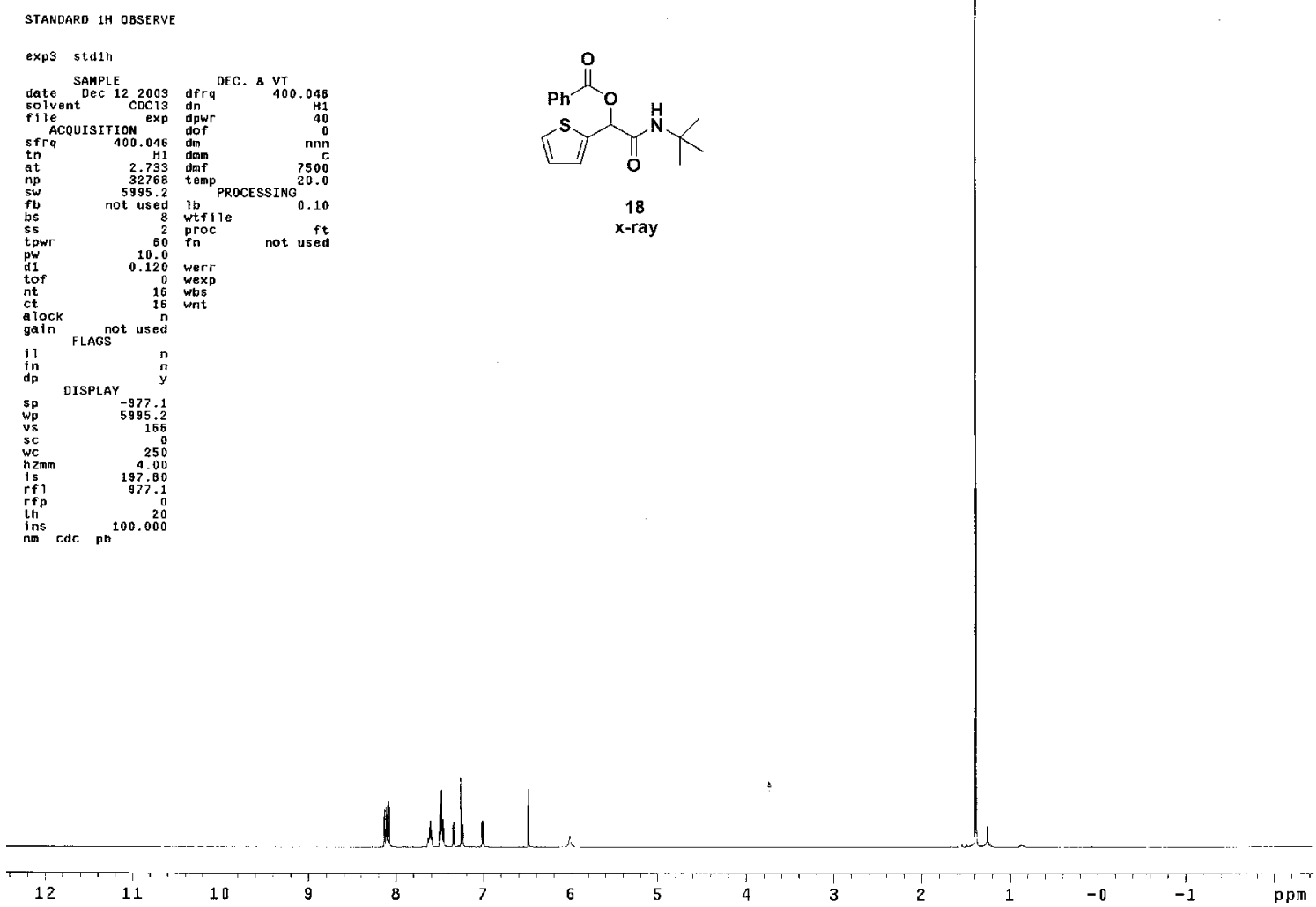

\section{Synthesis of 1-(butylcarbamoyl)-2-(benzyloxy)ethyl benzoate (19)}<smiles>CCCCNC(=O)C(COc1ccccc1)OC(=O)c1ccccc1</smiles>

19
Following General Procedure II, a mixture of isocyanide $\mathbf{1 4}$ (45 mg, $0.54 \mathrm{mmol}, 1.0$ equiv), benzoic acid (5) (70 mg, 0.57 mmol, 1.05 equiv), aldehyde 6 (86 mg, $91 \mu \mathrm{L}, 0.57 \mathrm{mmol}$, 1.05 equiv) and $20 \mathrm{~mol} \%$ of catalyst 4 in $\mathrm{CH}_{2} \mathrm{Cl}_{2}$ was stirred 
for 24 hrs. After removal of solvent followed by silica gel chromatography, 19 (167 mg, 87\%) was obtained as a brownish-white solid.

\section{Data for 19:}

${ }^{1}$ H NMR: $\quad\left(400 \mathrm{MHz}, \mathrm{CDCl}_{3}\right)$

$\delta$ 8.13-8.07 (m, 3H, aryl); 7.64-7.60 (m, 1H, aryl); 7.51-7.46 (m, 4H, aryl);

7.30-7.27 (m, 2H, aryl); 6.26 (bt, 1H, NH); 5.61 (t, $J=3.6 \mathrm{~Hz}, 1 \mathrm{H}, \mathrm{CH}_{2}$ );

4.59 (s, 2H, $\mathrm{CH}_{2}$ ); 4.04-4.00 (dd, $J=4.8,10.8 \mathrm{~Hz}, 1 \mathrm{H}, \mathrm{CH}$ ); 3.95-3.91 (dd, $J=3.6,10.8 \mathrm{~Hz}, 1 \mathrm{H}, \mathrm{CH}) ; 3.38-3.26\left(\mathrm{~m}, 2 \mathrm{H}, \mathrm{CH}_{2}\right) ; 1.53-1.45(\mathrm{q}, J=6.8$

$\left.\mathrm{Hz}, 2 \mathrm{H}, \mathrm{CH}_{2}\right) ; 1.36-1.27$ (m, 2H, $\left.\mathrm{CH}_{2}\right) ; 0.87$ (t, $J=7.2 \mathrm{~Hz}, 3 \mathrm{H}, \mathrm{CH}_{3}$ )

${ }^{13}$ C NMR: $\quad\left(100 \mathrm{MHz}, \mathrm{CDCl}_{3}\right)$

$\delta 168.0,165.4,138.9,134.0,130.4,130.1,129.5,128.9,128.7,127.9$, 73.8, 73.6, 69.7, 39.4, 31.8, 20.2, 13.9

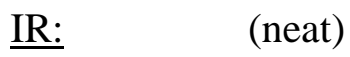

$v_{\max }=2929,1724,1690,1266,711 \mathrm{~cm}^{-1}$

TLC: $\quad \mathrm{Rf}=0.29$ (EtOAc:Hex 1:2)

HRMS: $\quad$ EIMS calculated for $\mathrm{C}_{21} \mathrm{H}_{25} \mathrm{NO}_{4}{ }^{+}[\mathrm{M}+\mathrm{H}]^{+}$: 355.1784, found: 355.1788

HPLC: $\quad(+/-)-19 t_{\mathrm{R}} 19.3 \min (49.2 \%) ; t_{\mathrm{R}} 23.7 \mathrm{~min}(50.8 \%)$

$(+)-19 t_{R} 18.9 \min (93.8 \%) ; t_{R} 23.1$ min (6.3\%) (Diacel Chiralpak AS-H $0.46 \mathrm{~cm} \phi \times 25 \mathrm{~cm}, 10 \%$ IPA in Hexane, $1 \mathrm{~mL} / \mathrm{min}$; isocratic)

OR: $\quad[\alpha]_{\mathrm{D}}^{22}=+7.7^{\circ}\left(\mathrm{CHCl}_{3}, \mathrm{c}=1.05\right)$ 


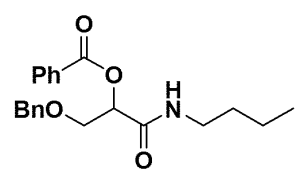

19

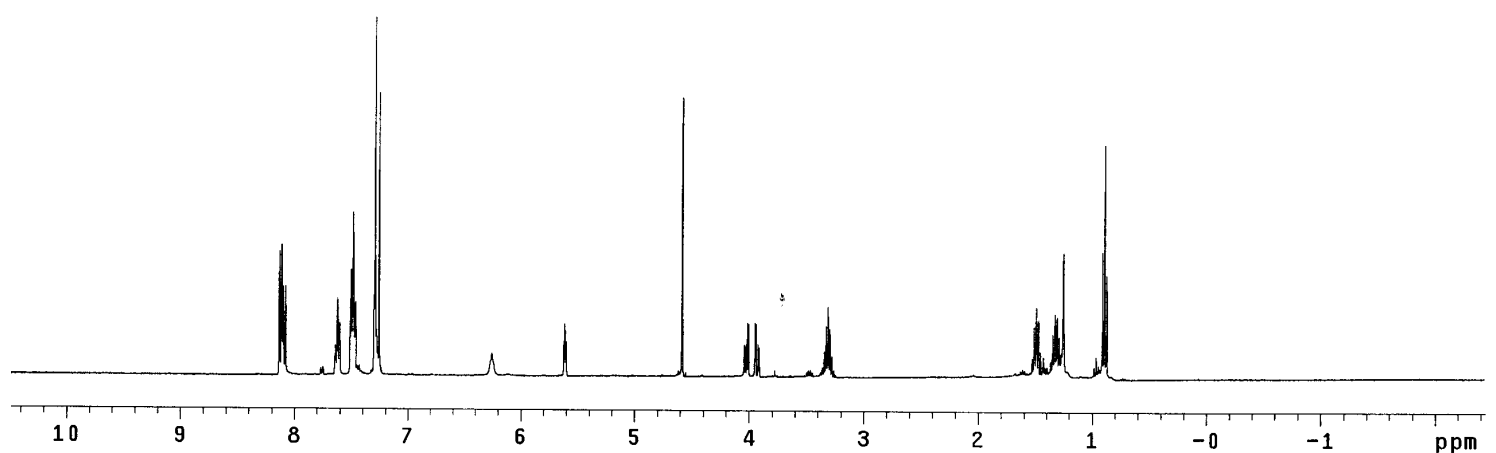

Synthesis of 1-(pentylcarbamoyl)-2-(benzyloxy)ethyl benzoate (20)<smiles>CCCCCNC(=O)C(COc1ccccc1)OC(=O)c1ccccc1</smiles>

20

Following General Procedure II, a mixture of isocyanide $\mathbf{1 5}$ (52 mg, $0.54 \mathrm{mmol}, 1.0$ equiv), benzoic acid (5) (70 mg, 0.57 mmol, 1.05 equiv), aldehyde 6 (86 mg, $91 \mu \mathrm{L}, 0.57$ mmol, 1.05 equiv) and $20 \mathrm{~mol} \%$ of catalyst 4 in $\mathrm{CH}_{2} \mathrm{Cl}_{2}$

was stirred for 24 hrs. After removal of solvent followed by silica gel chromatography, 20 (165 mg, 83\%) was obtained as a yellowish-white solid.

Data for 20:

${ }^{1} \mathrm{H}$ NMR: $\quad\left(400 \mathrm{MHz}, \mathrm{CDCl}_{3}\right)$

$\delta 8.08$ (dd, $J=1.2,8.0 \mathrm{~Hz}, 2 \mathrm{H}$, aryl); 7.64-7.60 (m, 2H, aryl); 7.51-7.47 (t, $J=8.0 \mathrm{~Hz}, 4 \mathrm{H}$, aryl); 7.30-7.27 (m, 2H, aryl); 6.24 (bt, 1H, NH); 5.60 (t, $J$ $\left.=3.2 \mathrm{~Hz}, 1 \mathrm{H}, \mathrm{CH}_{2}\right) ; 4.58$ (s, 2H, CH); 4.04-4.0 (dd, $J=5.2,11.2 \mathrm{~Hz}, 1 \mathrm{H}$, $\mathrm{CH}_{2}$ ); 3.95-3.91 (dd, $\left.J=3.6,11.2 \mathrm{~Hz}, 1 \mathrm{H}, \mathrm{CH}_{2}\right)$; 3.33-3.26 (m, $\left.2 \mathrm{H}, \mathrm{CH}_{2}\right)$; 1.54-1.47 (q, $\left.J=7.2 \mathrm{~Hz}, 2 \mathrm{H}, \mathrm{CH}_{2}\right) ; 1.33-1.23\left(\mathrm{~m}, 4 \mathrm{H}, \mathrm{CH}_{2}\right) ; 0.83(\mathrm{t}, J=6.8$ 
$\left.\mathrm{Hz}, 3 \mathrm{H}, \mathrm{CH}_{3}\right)$

${ }^{13} \underline{\mathrm{C} \mathrm{NMR}}: \quad\left(100 \mathrm{MHz}, \mathrm{CDCl}_{3}\right)$

$\delta$ 167.9, 165.5, 138.9, 133.9, 130.1, 129.5, 128.9, 128.6, 128.0, 127.8, 73.8, 73.6, 69.6, 39.6, 29.4, 29.2, 22.5, 14.2

IR: $\quad$ (neat)

$v_{\max }=2930,2860,1726,1663,1540,1452,1267,1097,712 \mathrm{~cm}^{-1}$

TLC: $\quad \mathrm{R} f=0.34$ (EtOAc:Hex 1:2)

HRMS: $\quad$ EIMS calculated for $\mathrm{C}_{22} \mathrm{H}_{27} \mathrm{NO}_{4}{ }^{+}[\mathrm{M}+\mathrm{H}]^{+}:$370.2019, found: 370.2013

HPLC: $\quad(+/-)-20 t_{\mathrm{R}} 28.2 \min (49.8 \%) ; t_{\mathrm{R}} 30.5 \min (50.2 \%)$

(+)-20 $t_{\mathrm{R}} 27.4 \min (94.7 \%) ; t_{\mathrm{R}} 31.2 \mathrm{~min}$ (5.3\%) (Diacel Chiralcel OJ 0.46

$\mathrm{cm} \phi$ x $25 \mathrm{~cm}$, Gradient; $t_{0} 0 \%$ IPA in Hexane, $t_{10 \min } \%$ IPA in Hexane, $t_{20 \min } 4 \%$ IPA in Hexane, $t_{30 \min } 6 \%$ IPA in Hexane, $1 \mathrm{~mL} / \mathrm{min}$ )

OR: $\quad[\alpha]_{\mathrm{D}}^{22}=+22.1^{\mathrm{o}}\left(\mathrm{CHCl}_{3}, \mathrm{c}=4.74\right)$ 


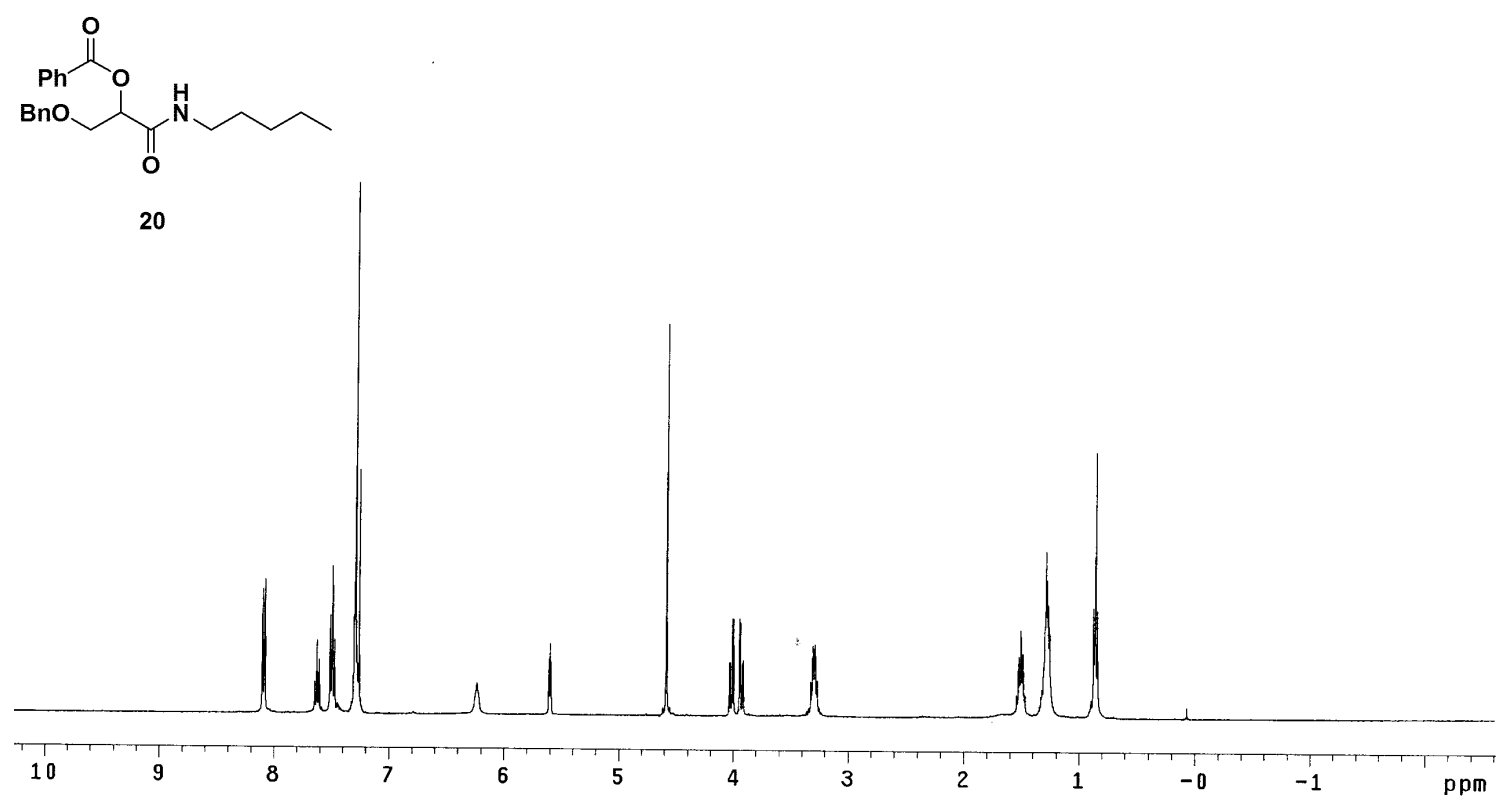

Synthesis of 1-(benzylcarbamoyl)-2-(benzyloxy)ethyl benzoate (21)<smiles>O=C(OC(COc1ccccc1)C(=O)NCc1ccccc1)c1ccccc1</smiles>

Following General Procedure II, a mixture of isocyanide 11 (58 mg, 0.5 mmol, 1.0 equiv), benzoic acid (5) (64 mg, $0.53 \mathrm{mmol}$, 1.05 equiv), aldehyde 6 (79 mg, $84 \mu \mathrm{L}, 0.53 \mathrm{mmol}, 1.05$ equiv) 21 and $20 \mathrm{~mol} \%$ of catalyst 4 in $\mathrm{CH}_{2} \mathrm{Cl}_{2}$ was stirred for 24 hrs. After removal of solvent followed by silica gel chromatography, 21 (173 mg, 89\%) was obtained as a brownish -white solid.

\section{Data for 21:}

${ }^{1} \mathrm{H}$ NMR: $\quad\left(400 \mathrm{MHz}, \mathrm{CDCl}_{3}\right)$

$\delta 8.12$ (d, $J=8 \mathrm{~Hz}, 2 \mathrm{H}$, aryl); 8.06 (dd, $J=1.2 \mathrm{~Hz}, 2 \mathrm{H}$, aryl); 7.64-7.58 (m, 4H, aryl); 7.51-7.45 (m, 4H, aryl); 7.35-7.28 (m, 3H, aryl); 6.60 (bt, $1 \mathrm{H}, \mathrm{NH}) ; 5.67$ (t, $J=4.0,1 \mathrm{H}, \mathrm{CH}) ; 4.64-4.59$ (dd, $1 \mathrm{H}, \mathrm{J}=6.4,15.2 \mathrm{~Hz}$, $\left.1 \mathrm{H}, \mathrm{CH}_{2}\right) ; 4.60$ (s, 2H, $\left.\mathrm{CH}_{2}\right) ; 4.48-4.43$ (dd, $J=5.2,16 \mathrm{~Hz}, 1 \mathrm{H}, \mathrm{CH}_{2}$ ); 4.11-4.07 (dd, $J=4.4,10.8 \mathrm{~Hz}, 1 \mathrm{H}, \mathrm{CH}_{2}$ ); 3.98-3.94 (dd, $J=3.6,10.4 \mathrm{~Hz}$, 
$1 \mathrm{H}, \mathrm{CH}_{2}$ )

${ }^{13} \underline{\mathrm{C} \mathrm{NMR}}: \quad\left(100 \mathrm{MHz}, \mathrm{CDCl}_{3}\right)$

$\delta$ 168.2, 165.5, 137.9, 137.8, 134.0, 130.5, 130.1, 129.1, 128.9, 128.9, $128.7,128.0,127.9,127.7,73.9,73.7,69.7,43.5$

IR: $\quad$ (neat)

$v_{\max }=1723,1691,1452,1265,710 \mathrm{~cm}^{-1}$

TLC: $\quad \mathrm{R} f=0.29$ (EtOAc:Hex 1:2)

HRMS: $\quad$ EIMS calculated for $\mathrm{C}_{24} \mathrm{H}_{23} \mathrm{NO}_{4}{ }^{+}[\mathrm{M}+\mathrm{H}]^{+}: 390.1706$, found: 390.1700

HPLC: $\quad(+/-)-21 t_{\mathrm{R}} 24.8 \min (49.3 \%) ; t_{\mathrm{R}} 28.0 \min (50.7 \%)$

$(+)-21 t_{\mathrm{R}} 24.2 \min (96.6 \%) ; t_{\mathrm{R}} 27.7 \mathrm{~min}(3.4 \%)$ (Chiralcel OD, 10\% IPA in Hexane, $1 \mathrm{~mL} / \mathrm{min}$ )
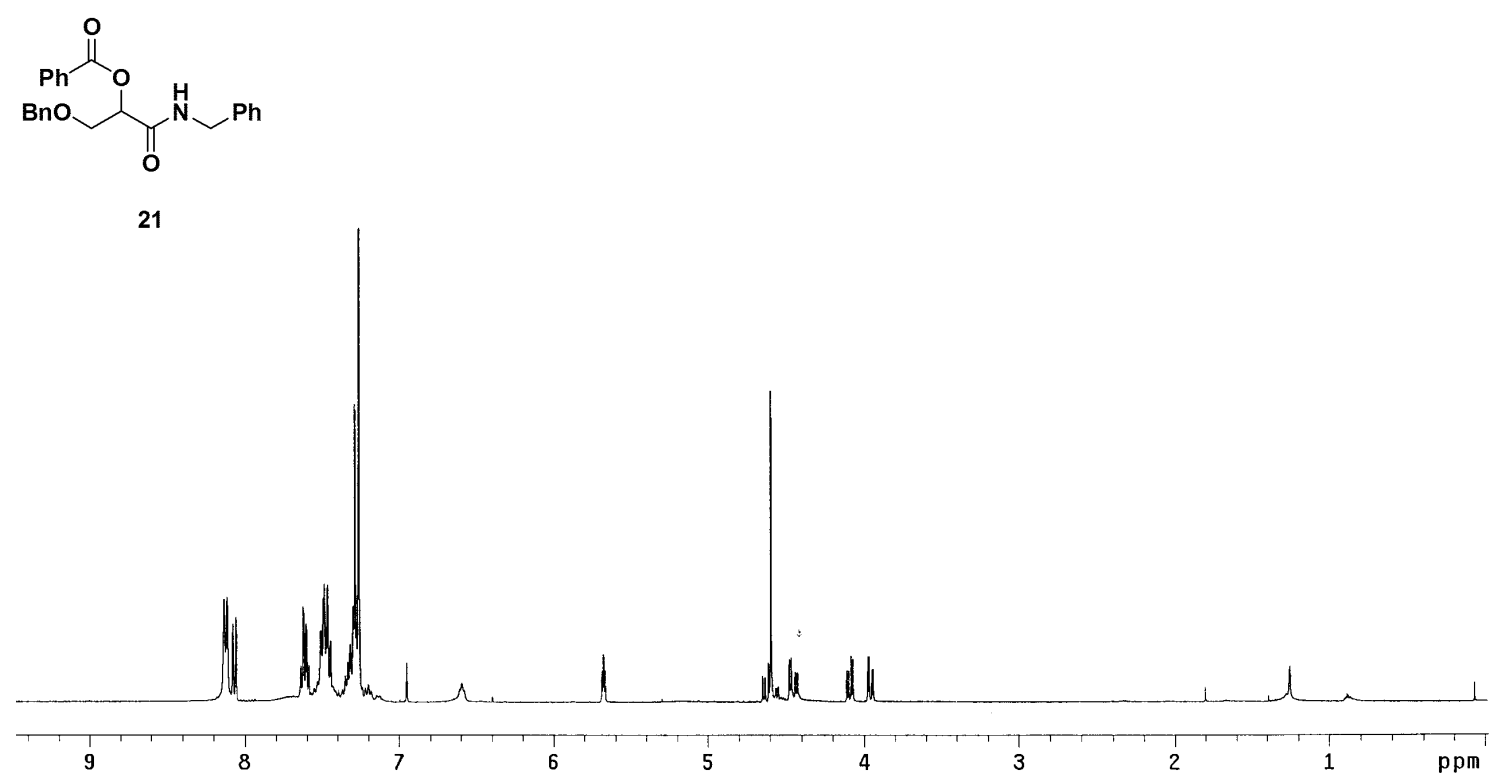

\section{Synthesis of 1-(tert-butylcarbamoyl)-2-(benzyloxy)ethyl benzoate (22)}<smiles>CC(C)(C)NC(=O)C(CO)OC(=O)c1ccccc1</smiles>

Following General Procedure II, a mixture of isocyanide 13 (42 mg, $58 \mu \mathrm{L}, 0.51 \mathrm{mmol}, 1.0$ equiv), benzoic acid (5) (65 mg, 0.54 
mmol, 1.05 equiv), aldehyde 6 ( $80 \mathrm{mg}, 86 \mu \mathrm{L}, 0.54 \mathrm{mmol}, 1.05$ equiv) and $20 \mathrm{~mol} \%$ of catalyst 4 in $\mathrm{CH}_{2} \mathrm{Cl}_{2}$ was stirred for 24 hrs. After removal of solvent followed by silica gel chromatography, 22 (181 mg, 95\%) was obtained as a whitish-brown solid.

Data for 22:

${ }^{1}$ H NMR: $\quad\left(400 \mathrm{MHz}, \mathrm{CDCl}_{3}\right)$

$\delta$ 8.10-8.07 (dd, $J=1.6,6.8 \mathrm{~Hz}, 2 \mathrm{H}$, aryl); 7.64-7.60 (tt, $J=1.2,7.6 \mathrm{~Hz}$, 2H, aryl); 7.50-7.46 (t, $J=8.0 \mathrm{~Hz}, 4 \mathrm{H}$, aryl); 7.30-7.27 (m, 2H, aryl); 6.10 (bs, $1 \mathrm{H}, \mathrm{NH}) ; 5.50$ (dd, $\left.J=1.6,3.2 \mathrm{~Hz}, 1 \mathrm{H}, \mathrm{CH}_{2}\right) ; 4.60(\mathrm{~d}, J=2.4 \mathrm{~Hz}, 2 \mathrm{H}$, $\mathrm{CH}_{2}$ ); 4.02-3.98 (dd, $J=4.4,10.4 \mathrm{~Hz}, 1 \mathrm{H}, \mathrm{CH}_{2}$ ); $3.93-3.90$ (dd, $J=4.0$, $\left.10.8 \mathrm{~Hz}, 1 \mathrm{H}, \mathrm{CH}_{2}\right) ; 1.36\left(\mathrm{~s}, 9 \mathrm{H}, \mathrm{CH}_{3}\right)$

${ }^{13} \mathrm{C}$ NMR: $\quad\left(100 \mathrm{MHz}, \mathrm{CDCl}_{3}\right)$ $\delta 167.1,165.4,140.8,138.0,134.0,130.4,130.1,128.9,128.7,127.8$, 73.9, 73.6, 69.7, 51.7, 28.9

IR: $\quad$ (neat)

$$
v_{\max }=1722,1688,1290,1264,709 \mathrm{~cm}^{-1}
$$

TLC: $\quad \mathrm{R} f=0.41($ EtOAc:Hex 1:2)

HRMS: $\quad$ EIMS calculated for $\mathrm{C}_{21} \mathrm{H}_{25} \mathrm{NO}_{4}{ }^{+}[\mathrm{M}+\mathrm{H}]^{+}: 356.1863$, found: 356.1855

HPLC: $\quad(+/-)-22 t_{\mathrm{R}} 20.7 \min (50.8 \%) ; t_{\mathrm{R}} 23.1 \min (49.2 \%)$

$(+)-22 t_{\mathrm{R}} 20.5 \min (98.8 \%) ; t_{\mathrm{R}} 23.3 \min (1.2 \%)$ (Diacel Chiralpak AS-H $0.46 \mathrm{~cm} \phi \times 25 \mathrm{~cm}, 2 \%$ IPA in Hexane, $0.7 \mathrm{~mL} / \mathrm{min}$; isocratic)

OR: $\quad[\alpha]_{\mathrm{D}}^{22}=+5.5^{\circ}\left(\mathrm{CHCl}_{3}, \mathrm{c}=2.12\right)$ 

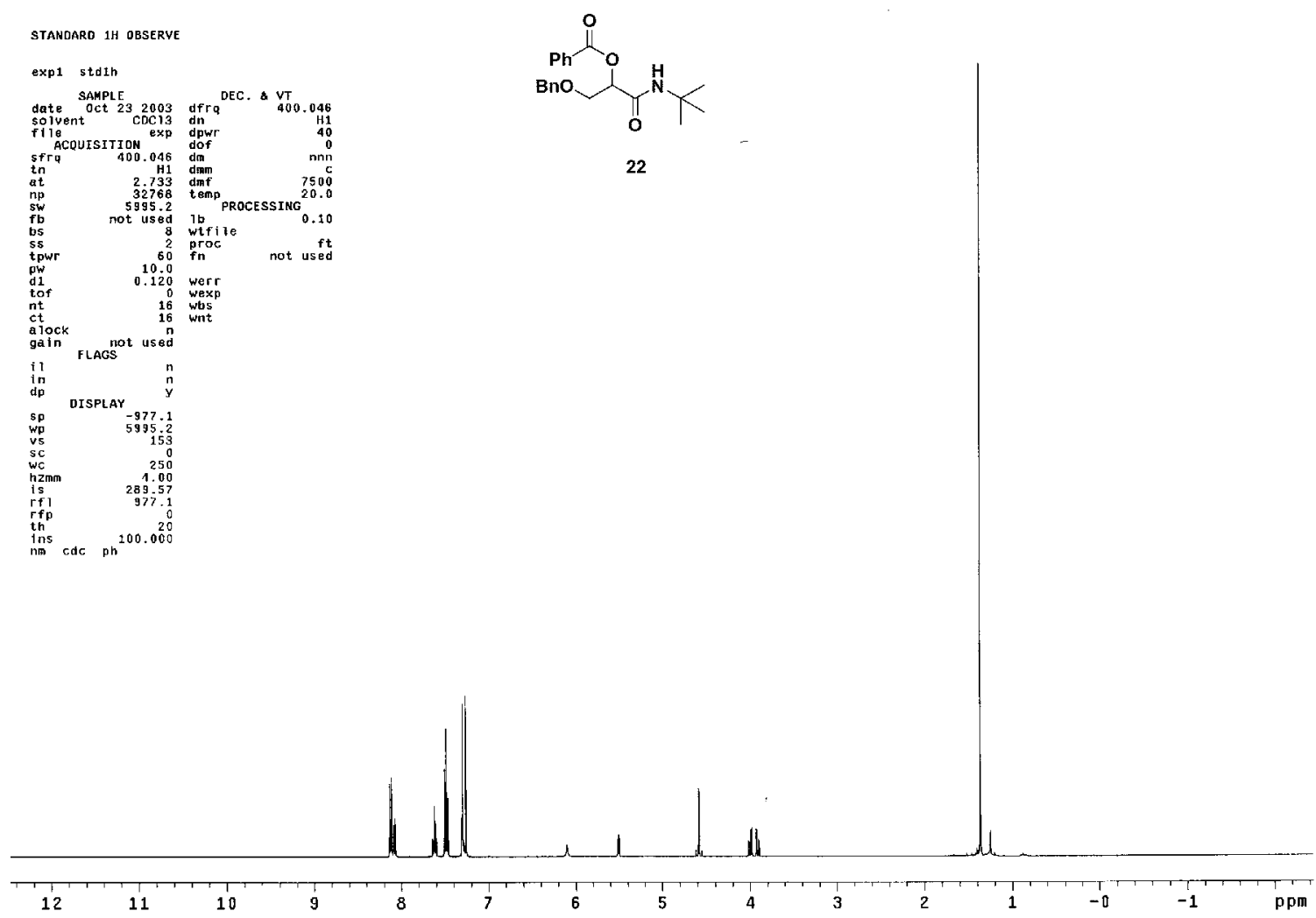

\section{Synthesis of (4-methoxyphenylcarbamoyl)(furan-2-yl)methyl benzoate (23)}

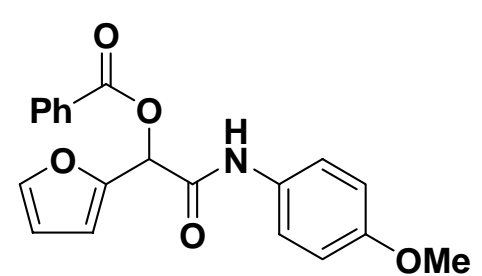

23

Following General Procedure II, a mixture of isocyanide 7 (67 mg, $0.50 \mathrm{mmol}, 1.0$ equiv), benzoic acid (5) (64 mg,

0.53 mmol, 1.05 equiv), aldehyde 10 (50 mg, $43 \mu \mathrm{L}, 0.53$ mmol, 1.05 equiv) and 20 mol \% of catalyst 4 in $\mathrm{CH}_{2} \mathrm{Cl}_{2}$

was stirred for 24 hrs. After removal of solvent followed by silica gel chromatography, 23 (172 mg, 95\%) was obtained as a whitish-brown solid.

\section{Data for 23:}

${ }^{1} \mathrm{H} \mathrm{NMR:} \quad\left(400 \mathrm{MHz}, \mathrm{CDCl}_{3}\right)$

$\delta$ 8.13-8.10 (m, 2H, aryl); 7.91 (bs, $1 \mathrm{H}, \mathrm{NH}) ; 7.64-7.60$ (m, 2H, aryl);

7.45-7.51 (m, 5H, aryl); 6.88-6.84 (m, 2H, aryl); 6.65 (d, $J=3.2 \mathrm{~Hz}, 1 \mathrm{H}$, 
aryl); $6.58(\mathrm{~s}, 1 \mathrm{H}, \mathrm{CH}) ; 6.41$ (dd, $J=1.6,2.0 \mathrm{~Hz}, 1 \mathrm{H}$, aryl); 3.79 (s, 3H, $\left.\mathrm{CH}_{3}\right)$

${ }^{13}$ C NMR: $\quad\left(100 \mathrm{MHz}, \mathrm{CDCl}_{3}\right)$

$\delta$ 165.0, 164.1, 157.1, 148.0, 144.1, 134.1, 130.4, 130.2, 128.9, 128.7, 122.3, 114.4, 112.0, 111.0, 69.6, 55.7

IR: $\quad$ (neat)

$$
v_{\max }=1723,1684,1512,1246,710 \mathrm{~cm}^{-1}
$$

TLC: $\quad \mathrm{R} f=0.32($ EtOAc:Hex 1:2)

HRMS: $\quad$ EIMS calculated for $\mathrm{C}_{20} \mathrm{H}_{17} \mathrm{NO}_{5}{ }^{+}[\mathrm{M}+\mathrm{H}]^{+}: 352.1186$, found: 352.1183

HPLC: $\quad(+/-)-23 t_{\mathrm{R}} 26.6 \min (49.5 \%) ; t_{\mathrm{R}} 30.5 \min (50.5 \%)$

$(-)-23 t_{\mathrm{R}} 26.7 \min (4.6 \%) ; t_{\mathrm{R}} 30.5 \min (95.4 \%)$ (Diacel Chiralcel OJ 0.46 $\mathrm{cm} \phi \times 25 \mathrm{~cm}, 5 \%$ IPA in Hexane, $1 \mathrm{~mL} / \mathrm{min}$; isocratic)

OR: $\quad[\alpha]_{\mathrm{D}}^{22}=-36.8^{\circ}\left(\mathrm{CHCl}_{3}, \mathrm{c}=7.35\right)$ 


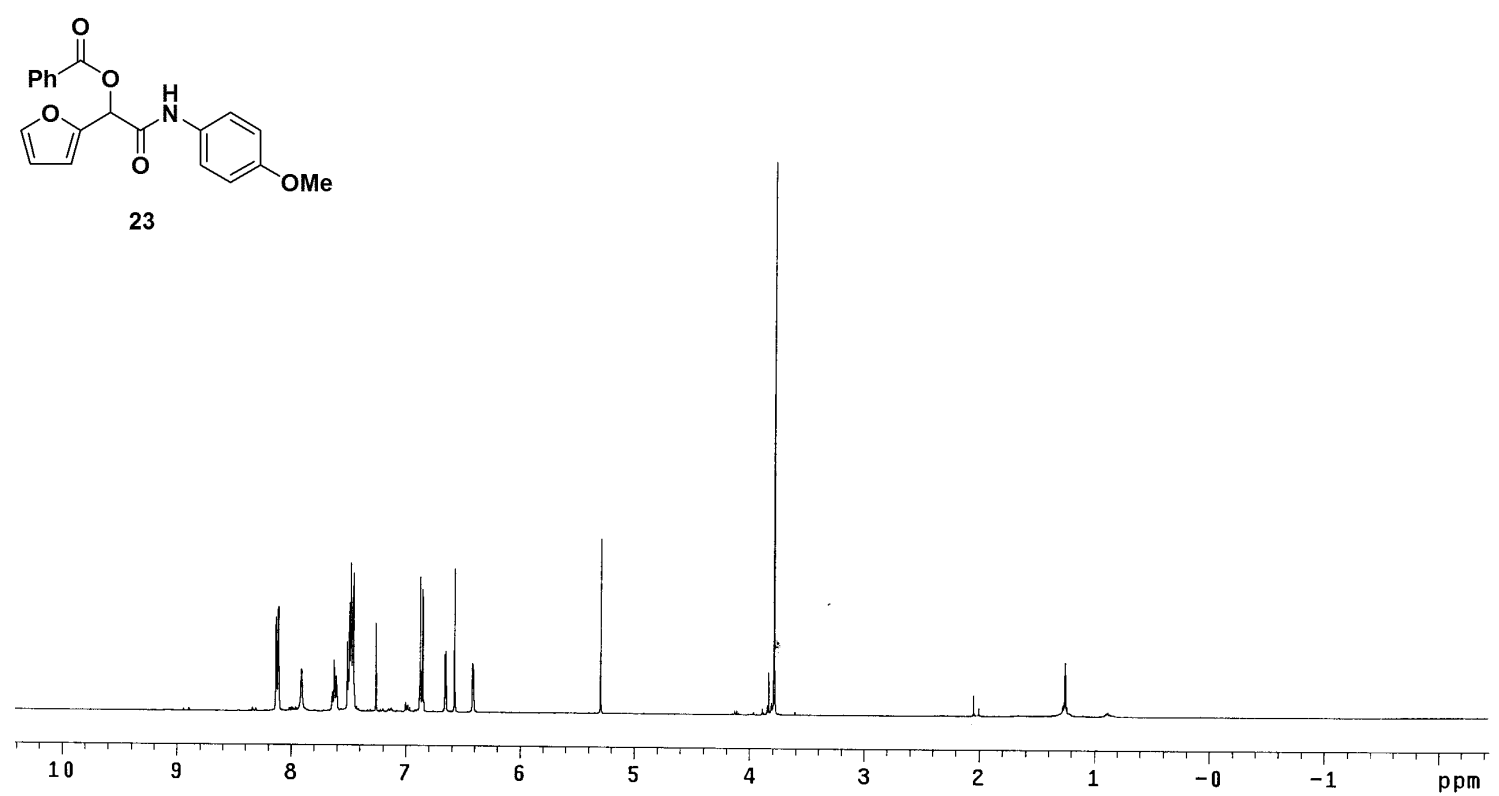

\section{Synthesis of (benzylcarbamoyl)(furan-2-yl)methyl benzoate (24)}<smiles>O=C(OC(C(=O)NCc1ccccc1)c1ccco1)c1ccccc1</smiles>

24

Following General Procedure II, a mixture of isocyanide 11 (53

mg, $55 \mu \mathrm{L}, 0.45$ mmol, 1.0 equiv), benzoic acid (5) (58 mg, 0.5 mmol, 1.05 equiv) and aldehyde 10 (45 mg, $39 \mu \mathrm{L}, 0.5 \mathrm{mmol}$, 1.05 equiv) in $\mathrm{CH}_{2} \mathrm{Cl}_{2}$ was stirred for 48 hrs. After removal of solvent and silica gel chromatography, 24 (135 mg, 90\%) was obtained as a white solid.

Data for 24:

${ }^{1}$ H NMR: $\quad\left(400 \mathrm{MHz}, \mathrm{CDCl}_{3}\right)$

$\delta$ 8.06-8.04 (dd, $J=1.2,8.4 \mathrm{~Hz}, 2 \mathrm{H}$, aryl); 7.61-7.56 (m, 1H, aryl); 7.497.42 (m, 4H, aryl); 7.35-7.25 (m, 4H, aryl); 6.63 (bm, 1H, NH); 6.59 (d, J $=3.2,1 \mathrm{H}$, aryl); 6.51 (s, $1 \mathrm{H}, \mathrm{CH}) ; 6.39$ (dd, $J=1.6, J=3.2,1 \mathrm{H}$, aryl); 4.64-4.58 (dd, $J=6.4,15.2 \mathrm{~Hz}, 1 \mathrm{H}, \mathrm{CH}_{2}$ ); 4.54-4.49 (dd, $J=6.0,15.2 \mathrm{~Hz}$, $1 \mathrm{H})$

${ }^{13}$ C NMR: $\quad\left(100 \mathrm{MHz}, \mathrm{CDCl}_{3}\right)$ 
$\delta$ 166.4, 165.1, 148.2, 143.9, 137.8, 134.0, 130.2, 129.1, 129.0, 128.8, 128.7, 127.8, 111.9, 111.0, 69.5, 43.7

IR: $\quad$ (neat)

$v_{\max }=1724,1670,1266,1093,1069,709 \mathrm{~cm}^{-1}$

TLC: $\quad \mathrm{R} f=0.36$ (EtOAc:Hex 1:2)

LRMS: $\quad$ LC/EIMS calculated for $\mathrm{C}_{20} \mathrm{H}_{17} \mathrm{NO}_{4}{ }^{+}[\mathrm{M}+\mathrm{H}]^{+}$: 336.1, found: 336.1

HPLC: $\quad(+/-)-24 t_{\mathrm{R}} 31.4 \min (50.1 \%) ; t_{\mathrm{R}} 39.9 \min (49.9 \%)$

(-)-24 $t_{\mathrm{R}} 32.8 \mathrm{~min}$ (12.4\%); $t_{\mathrm{R}} 40.3 \mathrm{~min}$ (87.6\%) (Diacel Chiralcel OD 0.46

$\mathrm{cm} \phi \times 25 \mathrm{~cm}$, 5\% IPA in Hexane, $1 \mathrm{~mL} / \mathrm{min}$; isocratic)

OR: $\quad[\alpha]_{\mathrm{D}}^{22}=-12.1^{\circ}\left(\mathrm{CHCl}_{3}, \mathrm{c}=1.57\right)$
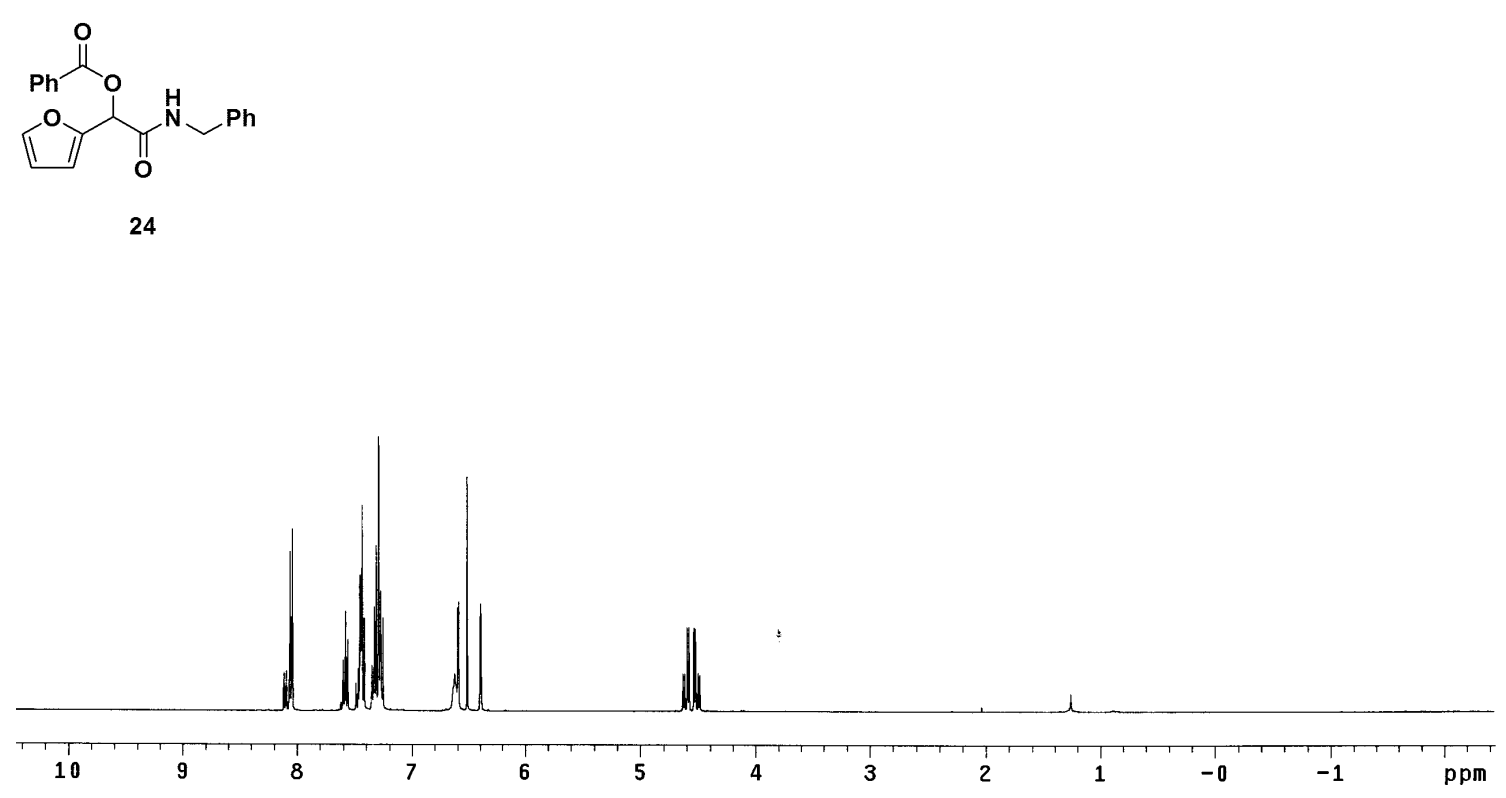

\section{Synthesis of (tert-butylcarbamoyl)(furan-2-yl)methyl benzoate (25)}<smiles>CC(C)(C)NC(=O)C(OC(=O)c1ccccc1)c1ccco1</smiles>

25

Following General Procedure II, a mixture of isocyanide $\mathbf{1 3}$ (40.6 mg, $55 \mu \mathrm{L}, 0.49$ mmol, 1.0 equiv), benzoic acid (5) (63 mg, 0.5 mmol, 1.05 equiv) and aldehyde $\mathbf{1 0}$ (49 mg, $43 \mu \mathrm{L}, 0.5 \mathrm{mmol}, 1.05$ 
equiv) in $\mathrm{CH}_{2} \mathrm{Cl}_{2}$ was stirred for 48 hrs. After removal of solvent and silica gel chromatography, 25 (143 mg, 97\%) was obtained as a whitish-brown solid.

\section{Data for 25:}

${ }^{1} \mathrm{H} \mathrm{NMR:} \quad\left(400 \mathrm{MHz}, \mathrm{CDCl}_{3}\right)$

$\delta$ 8.09-8.06 (m, 2H, aryl); 7.63-7.57 (m, 1H, aryl); 7.50-7.43 (m, 3H, aryl);

6.57 (d, $J=3.2 \mathrm{~Hz}, 1 \mathrm{H}$, aryl); 6.38 (dd, $J=1.6,3.2 \mathrm{~Hz}, 1 \mathrm{H}$, aryl); 6.35 (s, 1H, CH); 6.08 (bs, 1H, NH); 1.40 (s, 9H, $\left.\mathrm{CH}_{3}\right)$

${ }^{13} \mathrm{C} \mathrm{NMR:} \quad\left(100 \mathrm{MHz}, \mathrm{CDCl}_{3}\right)$

$\delta$ 171.9, 165.3, 148.7, 143.8, 133.9, 130.4, 130.1, 128.8, 111.5, 110.9, 69.6, 52.1, 28.9

IR: $\quad$ (neat)

$v_{\max }=1724,1692,1452,1258,1107,709 \mathrm{~cm}^{-1}$

TLC: $\quad \mathrm{R} f=0.54($ EtOAc:Hex 1:2)

LRMS: $\quad$ LC/EIMS calculated for $\mathrm{C}_{17} \mathrm{H}_{19} \mathrm{NO}_{4}{ }^{+}[\mathrm{M}+\mathrm{H}]^{+}:$302.1, found: 302.1

HPLC: $\quad(+/-)-25 t_{\mathrm{R}} 22.7 \min (50.7 \%) ; t_{\mathrm{R}} 27.9 \min (49.3 \%)$

(-)-25 $t_{\mathrm{R}} 23.0 \min (5.6 \%) ; t_{\mathrm{R}} 28.2 \min$ (94.6\%) (Diacel Chiralcel OJ 0.46 $\mathrm{cm} \phi$ x 25 cm, 3\% IPA in Hexane, $1 \mathrm{~mL} / \mathrm{min}$; isocratic)

OR: $\quad[\alpha]_{\mathrm{D}}^{22}=-22.4^{\mathrm{o}}\left(\mathrm{CHCl}_{3}, \mathrm{c}=1.17\right)$ 

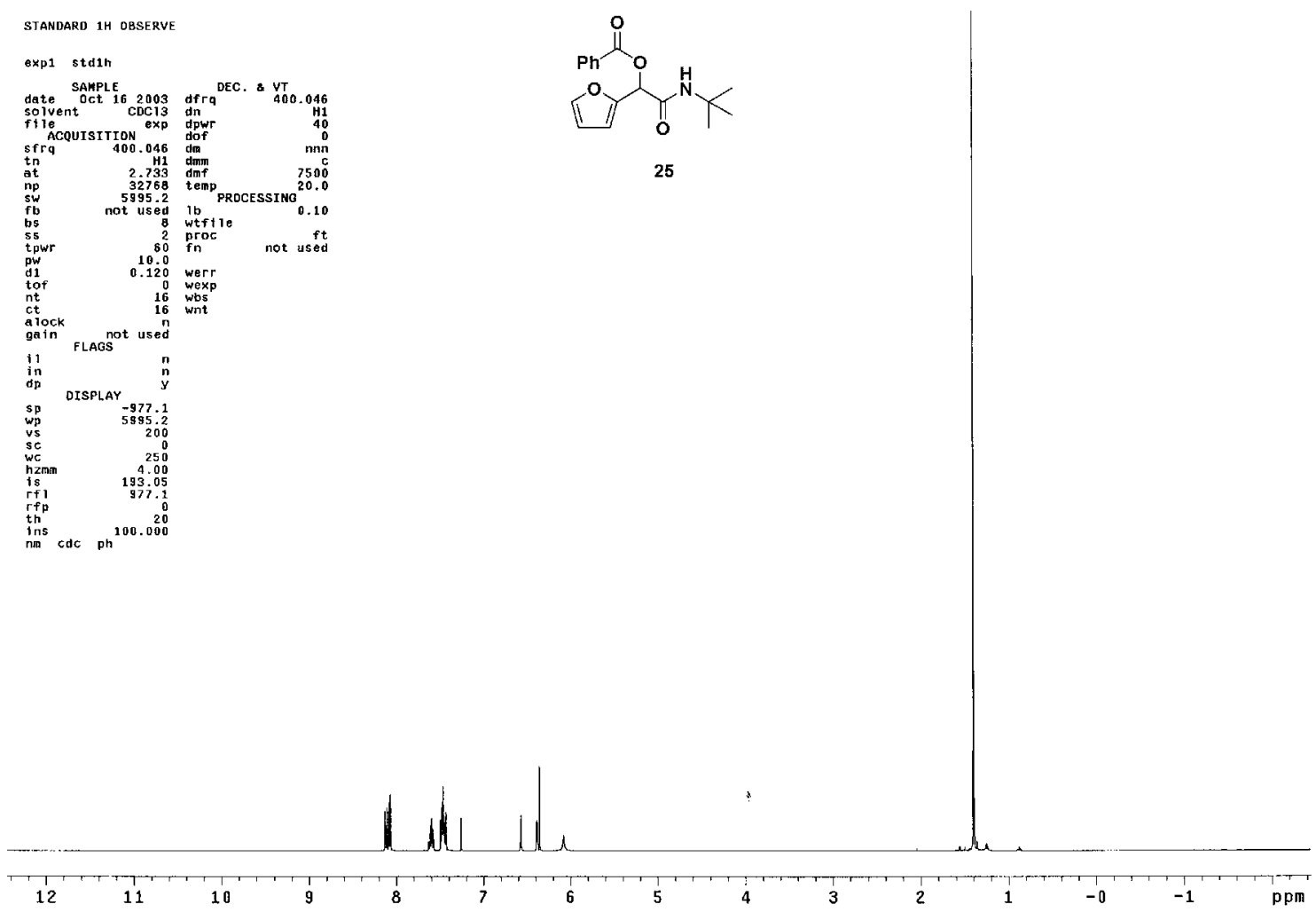

\section{Synthesis of (butylcarbamoyl)(furan-2-yl)methyl benzoate (26)}<smiles>CCCCNC(=O)C(OC(=O)c1ccccc1)c1ccco1</smiles>

26

Following General Procedure II, a mixture of isocyanide 14 (39 mg, $41 \mu \mathrm{L}, 0.47 \mathrm{mmol}, 1.0$ equiv), benzoic acid (5) (60 mg, 0.49 mmol, 1.05 equiv) and aldehyde 10 (47 mg, $41 \mu \mathrm{L}, 0.49$

mmol, 1.05 equiv) in $\mathrm{CH}_{2} \mathrm{Cl}_{2}$ was stirred for 48 hrs. After removal of solvent and silica gel chromatography, 26 (116 mg, 82\%) was obtained as a brownish solid.

\section{Data for 26:}

${ }^{1} \mathrm{H} \mathrm{NMR:} \quad\left(400 \mathrm{MHz}, \mathrm{CDCl}_{3}\right)$

$\delta$ 8.08-8.05 (dd, $J=0.8,4.4 \mathrm{~Hz}, 2 \mathrm{H}$, aryl); 7.59-7.55 (m, 1H, aryl); 7.45-

7.41 (m, 3H, aryl); 6.56 (d, $J=4.0 \mathrm{~Hz}, 1 \mathrm{H}$, aryl); 6.47-6.45 (bm, 1H, NH);

6.44 (s, $1 \mathrm{H}, \mathrm{CH}) ; 6.36$ (dd, $J=1.2, J=3.2,1 \mathrm{H}$, aryl); 3.38-3.28 (q, $J=6.0$ 
$\mathrm{Hz}, 2 \mathrm{H}, \mathrm{CH}_{2}$ ); $1.54-1.47$ (q, $J=7.6 \mathrm{~Hz}, 2 \mathrm{H}, \mathrm{CH}_{2}$ ); 1.36-1.29 (sext, $\mathrm{J}=7.6$

$\left.\mathrm{Hz}, 2 \mathrm{H}, \mathrm{CH}_{2}\right) ; 0.89\left(\mathrm{t}, J=7.2 \mathrm{~Hz}, 3 \mathrm{H}, \mathrm{CH}_{3}\right)$

${ }^{13} \mathrm{C}$ NMR: $\quad\left(100 \mathrm{MHz}, \mathrm{CDCl}_{3}\right)$

$\delta 166.4,165.1,148.4,143.8,133.9,130.1,129.2,128.8,111.6,110.9$, 69.5, 39.6, 31.7, 20.2, 13.9

IR: $\quad$ (neat)

$v_{\max }=3297,2958,1725,1668,1557,1265,1243,1107,707 \mathrm{~cm}^{-1}$

TLC: $\quad \mathrm{R} f=0.41$ (EtOAc:Hex 1:2)

HRMS: $\quad$ EIMS calculated for $\mathrm{C}_{17} \mathrm{H}_{19} \mathrm{NO}_{4}{ }^{+}[\mathrm{M}+\mathrm{H}]^{+}$: 302.1393 , found: 302.1356

HPLC: $\quad(+/-)-26 t_{\mathrm{R}} 21.9 \mathrm{~min}(52.2 \%) ; t_{\mathrm{R}} 27.9 \mathrm{~min}(47.8 \%)$

(-)-26 $t_{\mathrm{R}} 22.7 \mathrm{~min}(10.9 \%) ; t_{\mathrm{R}} 29.2 \mathrm{~min}$ (89.2\%) (Diacel Chiralcel OD 0.46

$\mathrm{cm} \phi \times 25 \mathrm{~cm}$, 5\% IPA in Hexane, $1 \mathrm{~mL} / \mathrm{min}$; isocratic)

OR: $\quad[\alpha]_{\mathrm{D}}^{22}=-8.7^{\circ}\left(\mathrm{CHCl}_{3}, \mathrm{c}=0.91\right)$ 


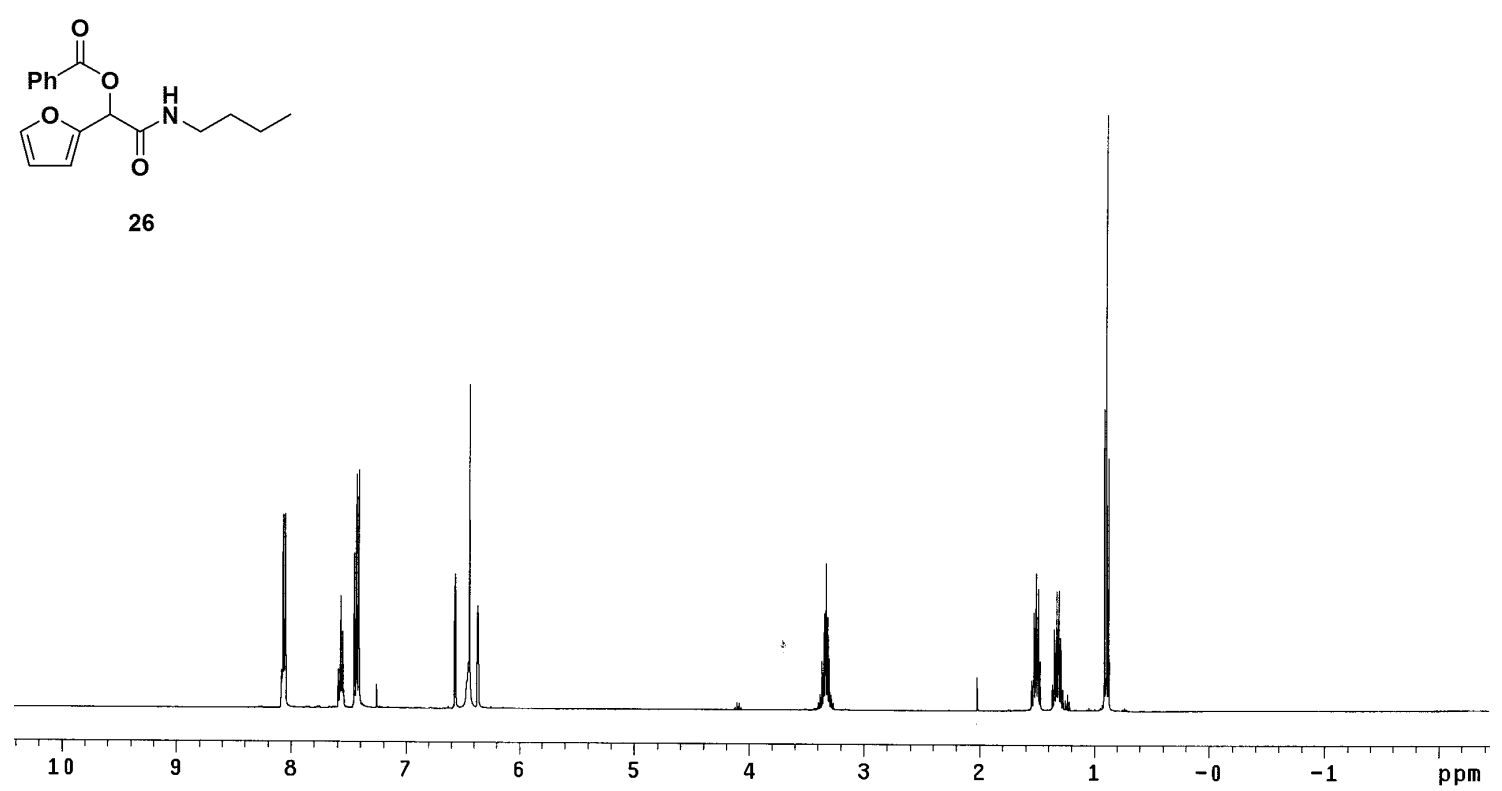

\section{Synthesis of (pentylcarbamoyl)(furan-2-yl)methyl benzoate (27)}

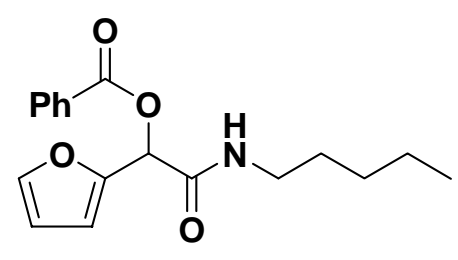

27
Following General Procedure II, a mixture of isocyanide $\mathbf{1 5}$ (44 mg, $57 \mu \mathrm{L}, 0.45$ mmol, 1.0 equiv), benzoic acid (5) (58 mg, 0.47 mmol, 1.05 equiv) and aldehyde 10 (45 mg, $39 \mu \mathrm{L}$, 0.47 mmol, 1.05 equiv) in $\mathrm{CH}_{2} \mathrm{Cl}_{2}$ was stirred for 48 hrs.

After removal of solvent and silica gel chromatography, 27 (116 mg, 82\%) was obtained as a whitish-brown solid.

\section{Data for 27:}

${ }^{1}$ H NMR: $\quad\left(400 \mathrm{MHz}, \mathrm{CDCl}_{3}\right)$

$\delta$ 8.09-8.06 (m, 2H, aryl); 7.62-7.57 (m, 1H, aryl); 7.48-7.42 (m, 3H, aryl);

$6.57(\mathrm{~d}, J=3.2 \mathrm{~Hz}, 1 \mathrm{H}$, aryl); 6.45 (s, $1 \mathrm{H}, \mathrm{CH}) ; 6.38(\mathrm{dd}, J=2.0,3.6 \mathrm{~Hz}$, 1H, aryl); 6.30 (bs, $1 \mathrm{H}, \mathrm{NH}$ ); 3.39-3.29 (sept, $J=7.2 \mathrm{~Hz}, 2 \mathrm{H}, \mathrm{CH}_{2}$ ); $1.58-$ 1.50 (quin, $J=7.2 \mathrm{~Hz}, 2 \mathrm{H}, \mathrm{CH}_{2}$ ); 1.34-1.24 (m, 4H, $\left.\mathrm{CH}_{2}\right) ; 0.86$ (t, $J=6.8$ $\left.\mathrm{Hz}, 3 \mathrm{H}, \mathrm{CH}_{3}\right)$ 
${ }^{13}$ C NMR: $\quad\left(100 \mathrm{MHz}, \mathrm{CDCl}_{3}\right)$

$\delta$ 166.2, 165.0, 148.3, 143.8, 134.0, 130.1, 129.2, 128.8, 111.7, 110.9,

69.5, 39.8, 29.3, 29.1, 22.5, 14.2

IR: $\quad$ (neat)

$v_{\max }=2932,1726,1665,1452,1265,710 \mathrm{~cm}^{-1}$

TLC: $\quad \mathrm{Rf}=0.47$ (EtOAc:Hex 1:2)

LRMS: $\quad$ EIMS calculated for $\mathrm{C}_{18} \mathrm{H}_{21} \mathrm{NO}_{4}{ }^{+}[\mathrm{M}+\mathrm{H}]^{+}$: 316.1, found: 316.2

HPLC: $\quad(+/-)-27 t_{\mathrm{R}} 15.0 \mathrm{~min}(53.1 \%) ; t_{\mathrm{R}} 19.8 \mathrm{~min}(46.9 \%)$

$(-)-27 t_{\mathrm{R}} 15.4 \mathrm{~min}(11.2 \%) ; t_{\mathrm{R}} 20.8 \mathrm{~min}$ (88.8\%) (Diacel Chiralcel OD 0.46 $\mathrm{cm} \phi \times 25 \mathrm{~cm}$, 5\% IPA in Hexane, $1 \mathrm{~mL} / \mathrm{min}$; isocratic)

OR: $\quad[\alpha]_{\mathrm{D}}^{22}=-37.3^{\circ}\left(\mathrm{CHCl}_{3}, \mathrm{c}=1.54\right)$
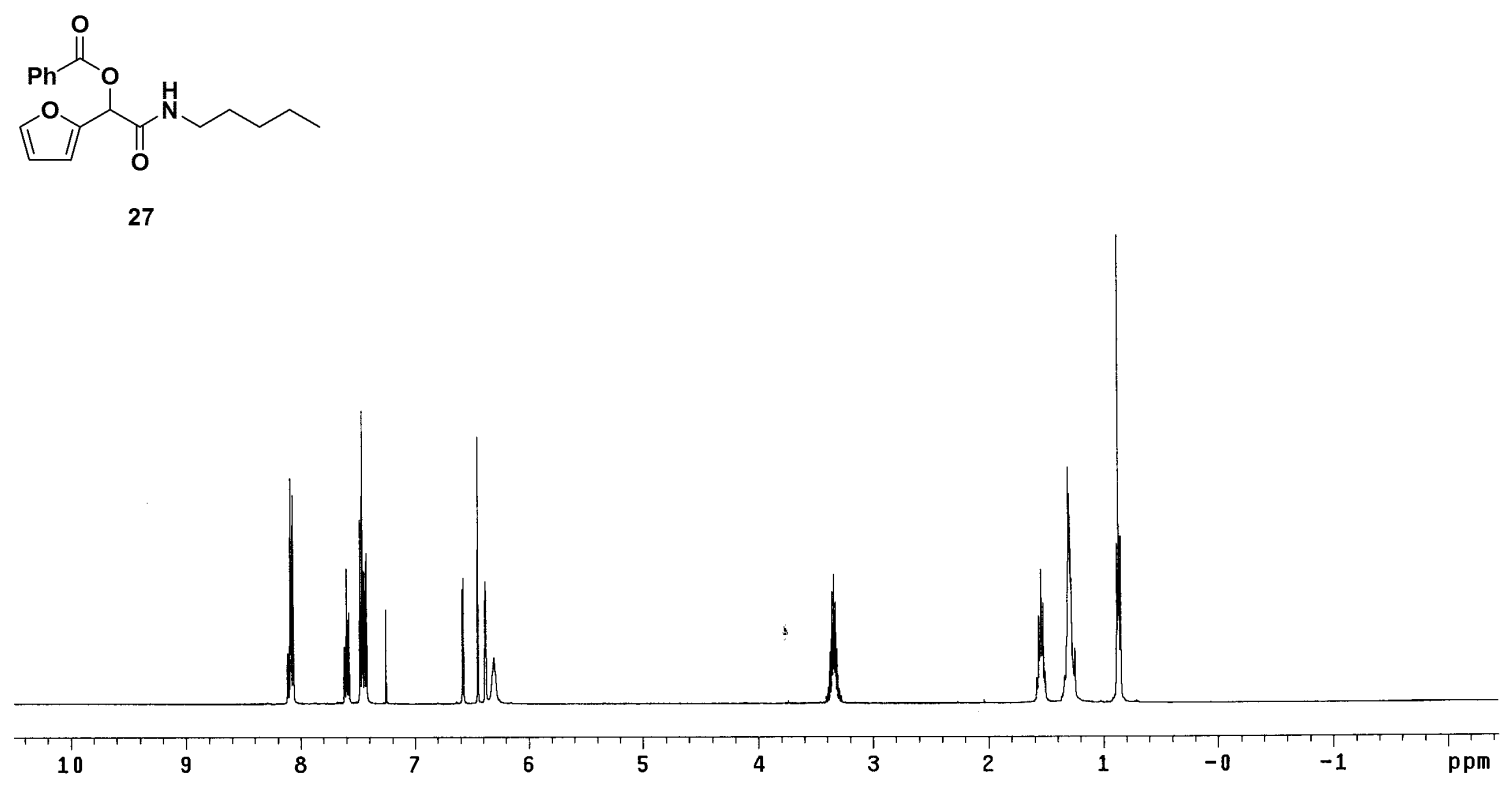


\section{Synthesis of (4-methoxyphenylcarbamoyl)(thiophen-2-yl)methyl benzoate (28)}

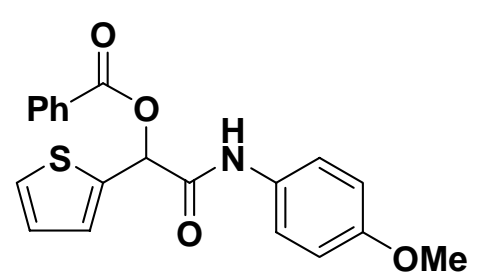

28
Following General Procedure II, a mixture of isocyanide 7 (60 mg, 0.45 mmol, 1.0 equiv), benzoic acid (5) (58 mg,

$0.47 \mathrm{mmol}, 1.05$ equiv) and aldehyde 12 (53 mg, $44 \mu \mathrm{L}$, 0.47 mmol, 1.05 equiv) in $\mathrm{CH}_{2} \mathrm{Cl}_{2}$ was stirred for 48 hrs.

After removal of solvent and silica gel chromatography, 28 (157 mg, 95\%) was obtained as a whitish-green solid.

Data for 28:

${ }^{1} \mathrm{H}$ NMR: $\quad\left(400 \mathrm{MHz}, \mathrm{CDCl}_{3}\right)$

$\delta$ 8.15-8.12 (dd, $J=0.8,8.4 \mathrm{~Hz}, 2 \mathrm{H}$, aryl); 7.83 (bs, 1H, NH); 7.65-7.61

(tt, $J=1.2,7.6 \mathrm{~Hz}, 1 \mathrm{H}$, aryl); 7.52-7.48 (m, 2H, aryl); 7.45-7.42 (m, 2H, aryl); 7.37 (dd, $J=0.8,5.2 \mathrm{~Hz}, 1 \mathrm{H}$, aryl); 7.32-7.31 (m, 1H, aryl); 7.02 (dd, $J=3.6,5.2,1 \mathrm{H}$, aryl); 6.86-6.84 (m, 2H, aryl); 6.72 (s, $1 \mathrm{H}, \mathrm{CH}) ; 3.78$ (s, $\left.3 \mathrm{H}, \mathrm{CH}_{3}\right)$

${ }^{13} \underline{\mathrm{C} \mathrm{NMR}}: \quad\left(100 \mathrm{MHz}, \mathrm{CDCl}_{3}\right)$ $\delta$ 165.5, 165.1, 157.2, 137.3, 134.1, 130.2, 130.0, 129.1, 129.0, 128.2, $127.4,127.3,122.3,114.4,72.1,55.7$

IR: $\quad$ (neat)

$v_{\max }=1724,1671,1512,1246,709 \mathrm{~cm}^{-1}$

TLC: $\quad \mathrm{R} f=0.29($ EtOAc:Hex 1:2)

HRMS: $\quad$ EIMS calculated for $\mathrm{C}_{20} \mathrm{H}_{17} \mathrm{NO}_{4} \mathrm{~S}^{+}[\mathrm{M}+\mathrm{H}]^{+}:$368.0957, found: 368.0915

HPLC: $\quad(+/-)-28 t_{\mathrm{R}} 30.9 \min (50.4 \%) ; t_{\mathrm{R}} 36.5 \min (49.6 \%)$

(-)-28 $t_{\mathrm{R}} 31.3 \min (5.7 \%) ; t_{\mathrm{R}} 38.1 \min$ (94.3\%) (Diacel Chiralcel OJ 0.46 
$\mathrm{cm} \phi \times 25 \mathrm{~cm}$, Gradient; $t_{0 \min } 15 \%$ IPA in Hexane, $t_{10 \min } 20 \%$ IPA in Hexane, $t_{20 \min } 25 \%$ IPA in Hexane, $t_{30 \min } 30 \%$ IPA in Hexane, $t_{40 \min } 35 \%$ IPA in Hexane, $t_{50 \min } 40 \%$ IPA in Hexane, $1 \mathrm{~mL} / \mathrm{min}$ )

OR: $\quad[\alpha]_{\mathrm{D}}^{22}=-6.9^{\circ}\left(\mathrm{CHCl}_{3}, \mathrm{c}=1.45\right)$
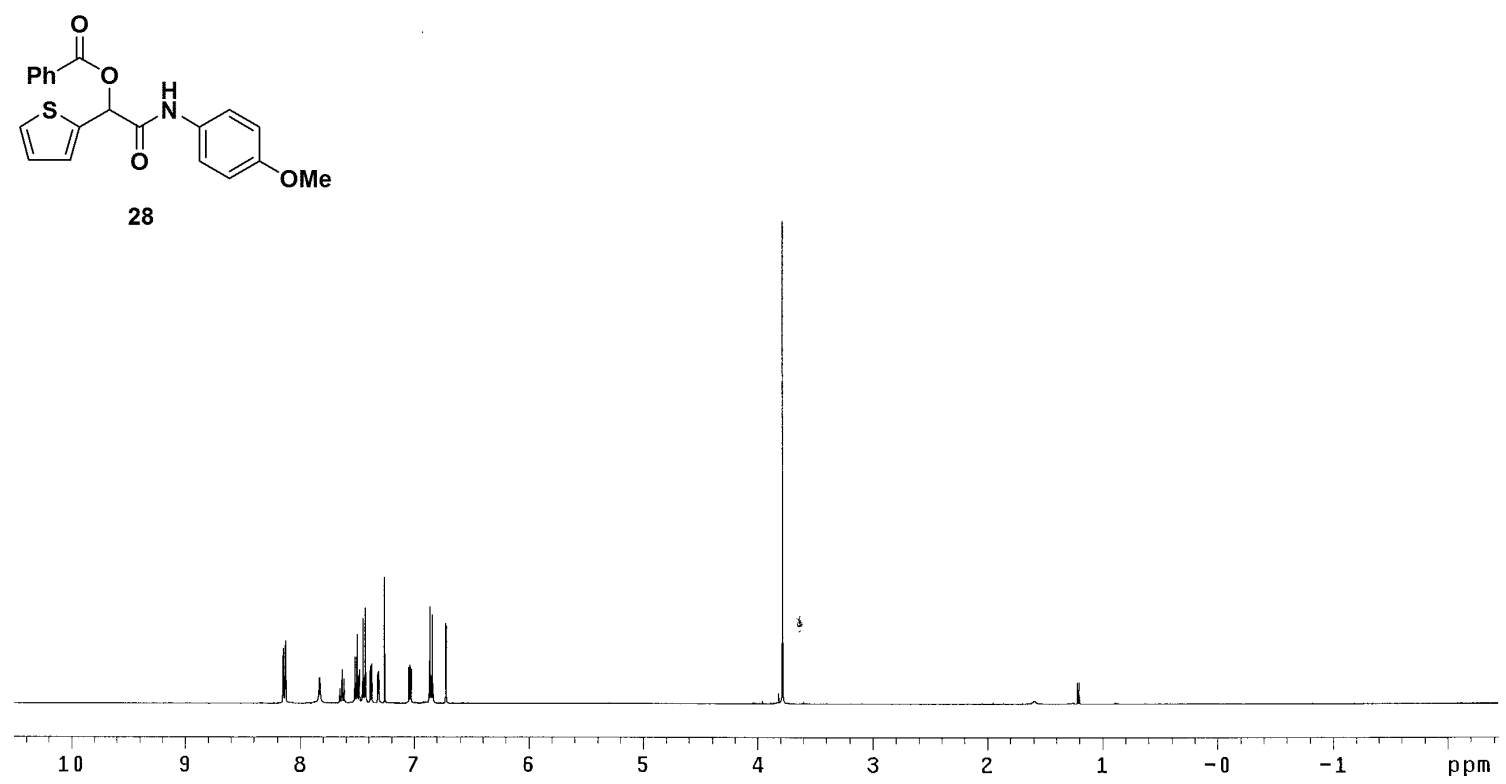

\section{Synthesis of (benzylcarbamoyl)(thiophen-2-yl)methyl benzoate (29)}<smiles>O=C(OC(C(=O)NCc1ccccc1)c1cccs1)c1ccccc1</smiles>

29

Following General Procedure II, a mixture of isocyanide 11 (53 mg, $55 \mu \mathrm{L}, 0.45$ mmol, 1.0 equiv), benzoic acid (5) (58 mg, 0.47 mmol, 1.05 equiv) and aldehyde 12 (53 mg, $43 \mu \mathrm{L}, 0.47 \mathrm{mmol}$, 1.05 equiv) in $\mathrm{CH}_{2} \mathrm{Cl}_{2}$ was stirred for 48 hrs. After removal of solvent and silica gel chromatography, 29 (137 mg, 87\%) was obtained as a whitish-gray solid.

Data for 29:

${ }^{1} \mathrm{H}$ NMR: $\quad\left(400 \mathrm{MHz}, \mathrm{CDCl}_{3}\right)$ $\delta$ 8.09-8.06 (dd, $J=1.2,8.0 \mathrm{~Hz}, 2 \mathrm{H}$, aryl); 7.62-7.57 (tt, $J=1.2,7.6 \mathrm{~Hz}$, 
1H, aryl); 7.48-7.42 (m, 2H, aryl); 7.37-7.26 (m, 7H, aryl); 7.03-7.01 (dd, $J$ = 3.2, $5.2 \mathrm{~Hz}, 1 \mathrm{H}$, aryl); 6.65 (s, 1H, CH); 6.55 (bt, 1H, NH); 4.60-4.55 (dd, $J=6.4,15.2 \mathrm{~Hz}, 1 \mathrm{H}, \mathrm{CH}_{2}$ ); 4.51-4.46 (dd, $J=5.2,14.4 \mathrm{~Hz}, 1 \mathrm{H}, \mathrm{CH}_{2}$ )

${ }^{13}$ C NMR: $\quad\left(100 \mathrm{MHz}, \mathrm{CDCl}_{3}\right)$ $\delta 167.7,165.2,137.8,137.5,134.0,130.2,129.1,129.0,128.9,128.7$, $128.2,127.9,127.9,127.2,72.0,43.7$

IR: $\quad$ (neat)

$v_{\max }=3300,1723,1667,1539,1452,1264,1107,707 \mathrm{~cm}^{-1}$

TLC: $\quad \mathrm{R} f=0.32($ EtOAc:Hex 1:2)

LRMS: $\quad$ EIMS calculated for $\mathrm{C}_{20} \mathrm{H}_{17} \mathrm{NO}_{3} \mathrm{~S}^{+}[\mathrm{M}+\mathrm{H}]^{+}:$352.1, found: 351.1

HPLC: $\quad(+/-)-29 t_{\mathrm{R}} 27.1 \min (46.5 \%) ; t_{\mathrm{R}} 30.3 \min (53.5 \%)$

$(-)-29 t_{\mathrm{R}} 27.7 \mathrm{~min}(12.7 \%) ; t_{\mathrm{R}} 30.2 \mathrm{~min}$ (87.3\%) (Diacel Chiralpak AS-H

$0.46 \mathrm{~cm} \phi \times 25 \mathrm{~cm}$, Gradient; $t_{0 \min } 10 \%$ IPA in Hexane, $t_{10 \min } 12 \%$ IPA in Hexane, $t_{20 \min } 14 \%$ IPA in Hexane, $t_{50 \min } 16 \%$ IPA in Hexane, $t_{65 \min } 20 \%$ IPA in Hexane, $1 \mathrm{~mL} / \mathrm{min}$ )

OR: $\quad[\alpha]_{\mathrm{D}}^{22}=-4.7^{\circ}\left(\mathrm{CHCl}_{3}, \mathrm{c}=3.4\right)$ 


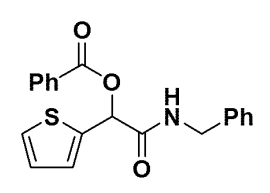

29

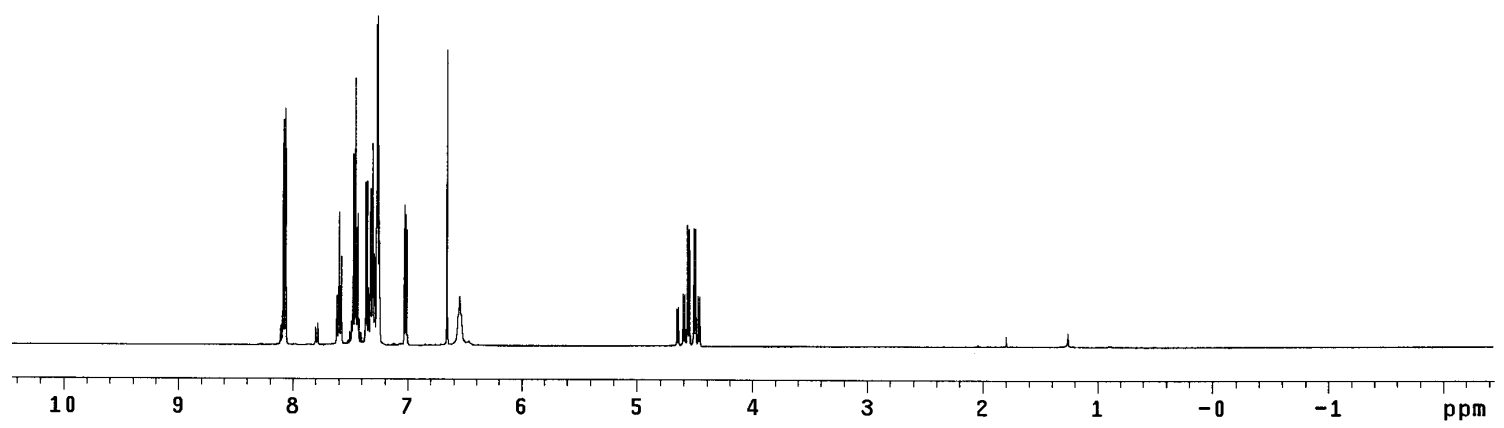

\section{Synthesis of (butylcarbamoyl)(thiophen-2-yl)methyl benzoate (30)}

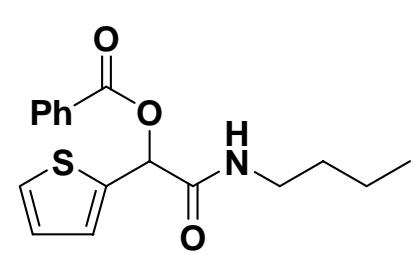

30

Following General Procedure II, a mixture of isocyanide $\mathbf{1 4}$ (40 mg, $41 \mu \mathrm{L}, 0.48 \mathrm{mmol}, 1.0$ equiv), benzoic acid (5) (61.5 mg, 0.50 mmol, 1.05 equiv) and aldehyde 12 (56 mg, $46 \mu \mathrm{L}$, 0.50 mmol, 1.05 equiv) in $\mathrm{CH}_{2} \mathrm{Cl}_{2}$ was stirred for 48 hrs. After removal of solvent and silica gel chromatography, 30 (116 mg, 76\%) was obtained as a whitish-green solid.

Data for 30:

${ }^{1}$ H NMR: $\quad\left(400 \mathrm{MHz}, \mathrm{CDCl}_{3}\right)$

$\delta$ 8.12-8.08 (m, 2H, aryl); 7.63-7.58 (m, 1H, aryl); 7.50-7.42 (m, 2H, aryl); 7.33 (dd, $J=1.2,5.2 \mathrm{~Hz}, 1 \mathrm{H}$, aryl); 7.25 (d, $J=4.0 \mathrm{~Hz}, 1 \mathrm{H}$, aryl); 7.00 (dd, $J=3.6,5.2 \mathrm{~Hz}, 1 \mathrm{H}$, aryl); 6.60 (s, 1H, CH); 6.27 (bt, 1H, NH); 3.40-3.26 (m, 2H, $\mathrm{CH}_{2}$ ); $1.55-1.47$ (quint, $J=7.6 \mathrm{~Hz}, 2 \mathrm{H}, \mathrm{CH}_{2}$ ); 1.37-1.29 (sext, $J=$ $\left.7.2 \mathrm{~Hz}, 2 \mathrm{H}, \mathrm{CH}_{2}\right) ; 0.88\left(\mathrm{t}, J=7.2 \mathrm{~Hz}, 3 \mathrm{H}, \mathrm{CH}_{3}\right)$ 
${ }^{13}$ C NMR: $\quad\left(100 \mathrm{MHz}, \mathrm{CDCl}_{3}\right)$

$\delta 167.7,165.1,137.7,134.0,130.4,130.2,129.3,128.8,128.0,127.1$, 71.9, 39.6, 31.7, 20.2, 13.9

IR: $\quad$ (neat)

$v_{\max }=3300,2958,2932,1725,1664,1543,1451,1264,1107,708 \mathrm{~cm}^{-1}$

TLC: $\quad \mathrm{Rf}=0.37$ (EtOAc:Hex 1:2)

LRMS: $\quad$ EIMS calculated for $\mathrm{C}_{17} \mathrm{H}_{19} \mathrm{NO}_{3} \mathrm{~S}^{+}[\mathrm{M}+\mathrm{H}]^{+}$: 318.1, found: 317.1

HPLC: $\quad(+/-)-30 t_{\mathrm{R}} 16.3 \min (48.2 \%) ; t_{\mathrm{R}} 20.6 \min (51.8 \%)$

$(-)-30 t_{R} 16.8 \min (18.2 \%) ; t_{R} 20.2 \min (81.7 \%)$ (Diacel Chiralpak AS-H

$0.46 \mathrm{~cm} \phi \times 25 \mathrm{~cm}$, Gradient; $t_{0 \min } 10 \%$ IPA in Hexane, $t_{10 \min } 12 \%$ IPA in Hexane, $t_{20 \min } 14 \%$ IPA in Hexane, $t_{50 \min } 16 \%$ IPA in Hexane, $t_{65 \min } 20 \%$ IPA in Hexane, $1 \mathrm{~mL} / \mathrm{min}$ )

OR: $\quad[\alpha]_{\mathrm{D}}^{22}=-5.6^{\circ}\left(\mathrm{CHCl}_{3}, \mathrm{c}=0.95\right)$
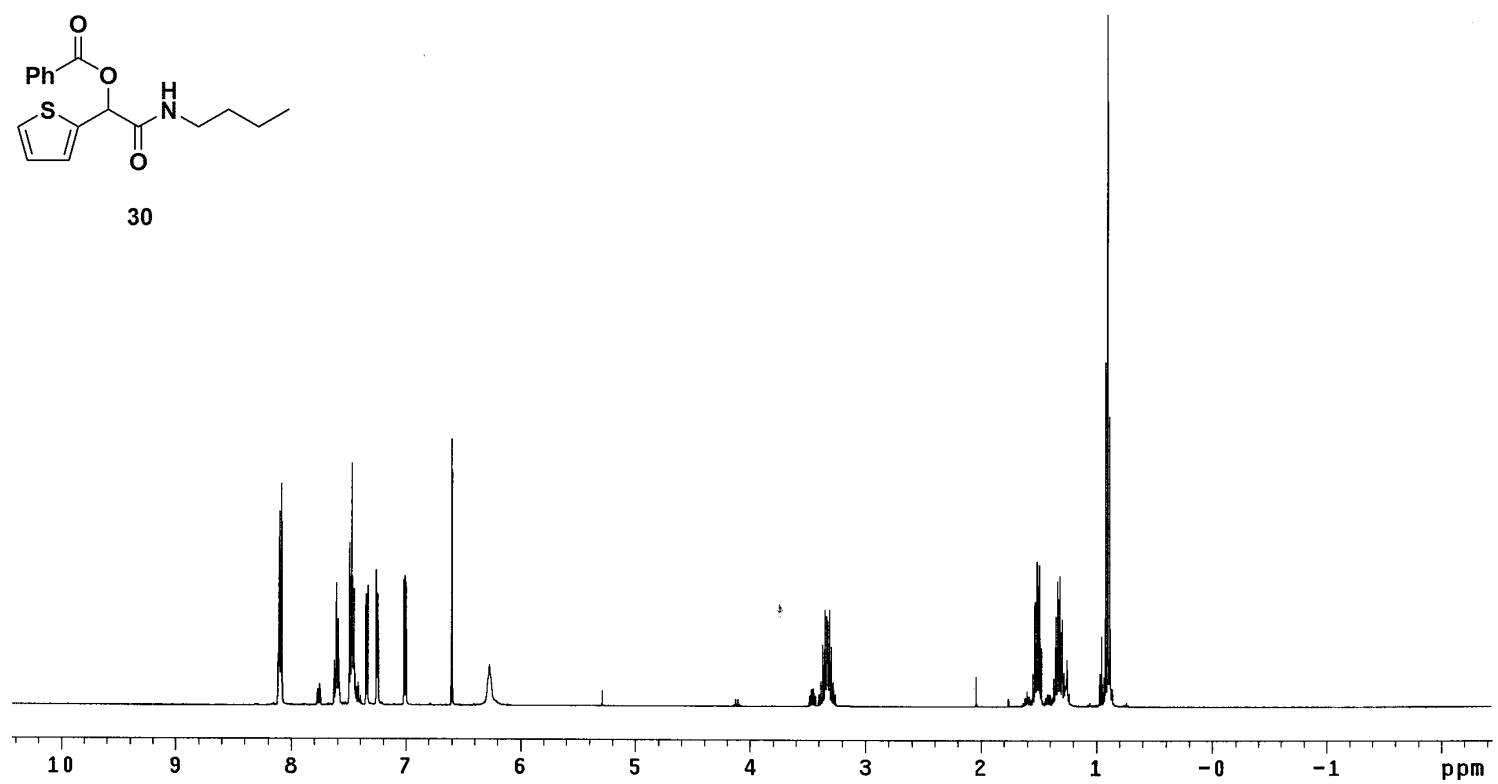


\section{Synthesis of (pentylcarbamoyl)(thiophen-2-yl)methyl benzoate (31)}

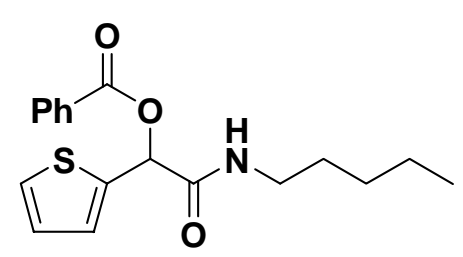

31

Following General Procedure II, a mixture of isocyanide $\mathbf{1 5}$ (44 mg, $57 \mu \mathrm{L}, 0.45$ mmol, 1.0 equiv), benzoic acid (5) (58 mg, 0.47 mmol, 1.05 equiv) and aldehyde 12 (53 mg, $43 \mu \mathrm{L}$, 0.47 mmol, 1.05 equiv) in $\mathrm{CH}_{2} \mathrm{Cl}_{2}$ was stirred for 48 hrs.

After removal of solvent and silica gel chromatography, 31 (112 mg, 75\%) was obtained as a whitish-brown solid.

\section{Data for 31:}

${ }^{1} \mathrm{H} \mathrm{NMR}: \quad\left(400 \mathrm{MHz}, \mathrm{CDCl}_{3}\right)$

$\delta$ 8.14-8.08 (m, 2H, aryl); 7.64-7.60 (m, 1H, aryl); $7.48(\mathrm{t}, J=8.0 \mathrm{~Hz}, 2 \mathrm{H}$, aryl); 7.34 (dd, $J=1.2,4.8 \mathrm{~Hz}, 1 \mathrm{H}$, aryl); 7.26 (m, 1H, aryl); 7.00 (dd, $J=$ 3.6, 4.8 Hz, 1H, aryl); 6.60 (s, 1H, CH); 6.21 (bt, 1H, NH); 3.40-3.26 (sept, $J=7.2 \mathrm{~Hz}, 2 \mathrm{H}, \mathrm{CH}_{2}$ ); 1.57-1.50 (quin, $J=6.8 \mathrm{~Hz}, 2 \mathrm{H}, \mathrm{CH}_{2}$ ); 1.36-1.23 (m, 4H, $\left.\mathrm{CH}_{2}\right) ; 0.85\left(\mathrm{t}, J=7.2 \mathrm{~Hz}, 3 \mathrm{H}, \mathrm{CH}_{3}\right)$

${ }^{13} \underline{\mathrm{C} \mathrm{NMR}}: \quad\left(100 \mathrm{MHz}, \mathrm{CDCl}_{3}\right)$ $\delta$ 167.6, 165.1, 137.7, 134.0, 130.4, 130.2, 129.5, 128.9, 128.3, 127.1, 71.9, 39.8, 29.3, 29.1, 22.5, 14.2

IR: $\quad$ (neat) $v_{\max }=3304,3072,2957,2931,2860,1724,1691,1451,1264,708 \mathrm{~cm}^{-1}$

TLC: $\quad \mathrm{R} f=0.42($ EtOAc:Hex 1:2)

LRMS: $\quad$ EIMS calculated for $\mathrm{C}_{18} \mathrm{H}_{21} \mathrm{NO}_{3} \mathrm{~S}^{+}[\mathrm{M}+\mathrm{H}]^{+}: 332.1$, found: 332.0

HPLC: $\quad(+/-)-31 t_{\mathrm{R}} 16.2 \min (48.0 \%) ; t_{\mathrm{R}} 19.0 \min (52.0 \%)$ 
(-)-31 $t_{\mathrm{R}} 17.3 \mathrm{~min}(20.0 \%) ; t_{\mathrm{R}} 22.4 \min (80.0 \%)$ (Diacel Chiralpak AS-H $0.46 \mathrm{~cm} \phi \times 25 \mathrm{~cm}, 10 \%$ IPA in Hexane, $1 \mathrm{~mL} / \mathrm{min}$; isocratic)

OR: $\quad[\alpha]_{\mathrm{D}}^{22}=-2.5^{\circ}\left(\mathrm{CHCl}_{3}, \mathrm{c}=1.41\right)$

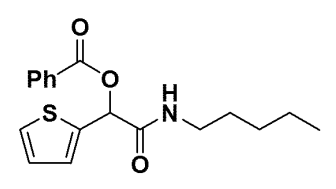

31

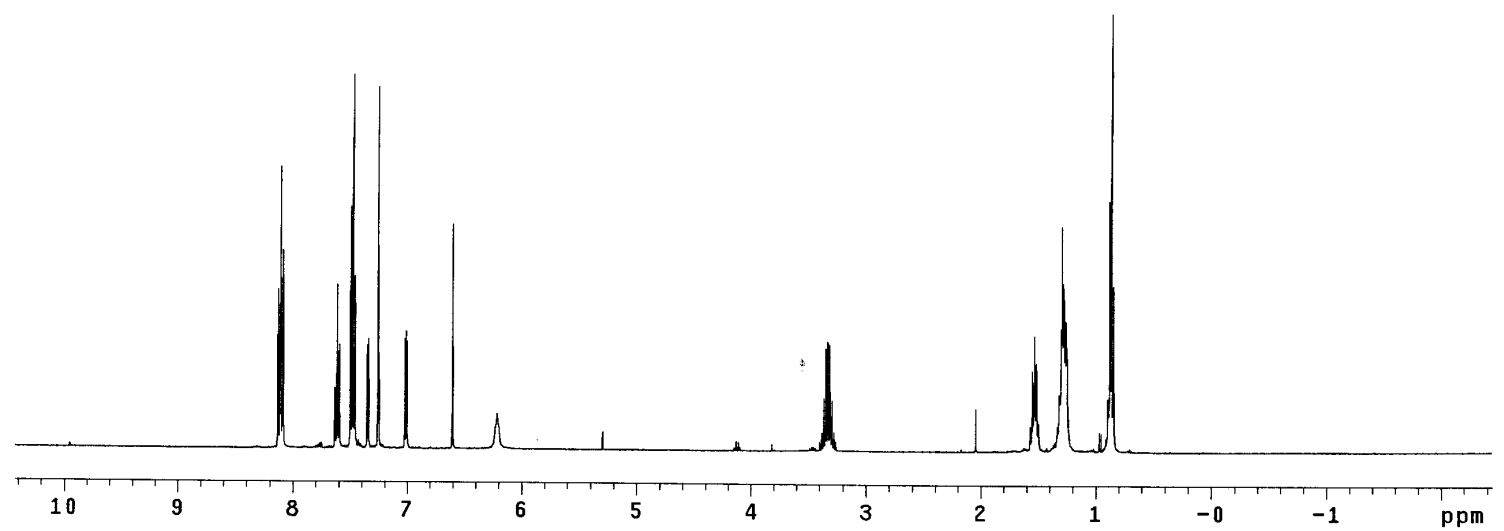

\section{Synthesis of tricyclic lactone (33)}

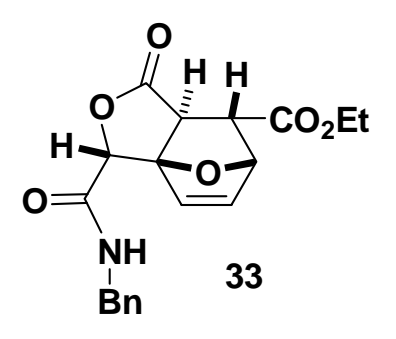

Following General Procedure II, a mixture of isocyanide 11 (27 mg, $28 \mu \mathrm{L}, 0.23 \mathrm{mmol}, 1.0$ equiv), fumaric acid monoethyl ester (32) (35 mg, $0.24 \mathrm{mmol}, 1.05$ equiv) and aldehyde 10 (23 mg, 20 $\mu \mathrm{L}, 0.24$ mmol, 1.05 equiv) in $\mathrm{CH}_{2} \mathrm{Cl}_{2}$ was stirred 48 hrs. After removal of solvent and silica gel chromatography, 33 (112 $\mathrm{mg}, 75 \%)$ was obtained as a yellowish-brown oil which was a mixture of diastereomers in 15:1 ratio as noted by ${ }^{1} \mathrm{H}$ NMR. The major diastereomer was isolated by silica gel chromatography.

\section{Data for 33:}

${ }^{1}$ H NMR: $\quad\left(500 \mathrm{MHz}, \mathrm{CDCl}_{3}\right)$ 
$\delta$ 7.34-7.25 (m, 5H, aryl); 6.80 (d, $J=6.0 \mathrm{~Hz}, 1 \mathrm{H}, \mathrm{CH}$ olefin); 6.77 (bt, 1H, NH); 6.40-6.39 (dd, $J=1.5,5.0 \mathrm{~Hz}, 1 \mathrm{H}, \mathrm{CH}$ olefin); 5.42 (s, 1H, CH); 5.35-5.34 (dd, $J=1.5,4.5 \mathrm{~Hz}, 1 \mathrm{H}$, CH oxygen bridge); 4.56-4.51 (dd, $J=$ 6.5, $15.5 \mathrm{~Hz}, 1 \mathrm{H}, \mathrm{CH}$ benzyl); 4.48-4.43 (dd, $J=6.0,15.0 \mathrm{~Hz}, 1 \mathrm{H}, \mathrm{CH}$ benzyl); 4.16-4.12 (q, $J=7.0 \mathrm{~Hz}, 2 \mathrm{H}, \mathrm{CH}_{2}$ ); 3.57-3.56 (dd, $J=3.0,5.0 \mathrm{~Hz}$, 1H, CH); 3.21 (d, $J=3.5 \mathrm{~Hz}, 1 \mathrm{H}, \mathrm{CH}) ; 1.26$ (t, $\left.J=8.0 \mathrm{~Hz}, \mathrm{CH}_{3}\right)$

${ }^{13}$ C NMR: $\quad\left(100 \mathrm{MHz}, \mathrm{CDCl}_{3}\right)$

$\delta 174.3,170.3,166.9,137.9,135.2,135.1,134.4,129.1,129.0,128.3$, 128.2, 128.1, 128.0, 92.1, 80.4, 63.5, 61.5, 50.7, 47.3, 46.3, 44.0, 14.4

TLC: $\quad \mathrm{R} f=0.37$ (EtOAc:Hex 1:2)

LRMS: $\quad$ EIMS calculated for $\mathrm{C}_{19} \mathrm{H}_{19} \mathrm{NO}_{6}{ }^{+}[\mathrm{M}+\mathrm{H}]^{+}$: 358.1, found: 358.0

HPLC: $\quad(+/-)-33 t_{\mathrm{R}} 25.2 \mathrm{~min}(47.9 \%) ; t_{\mathrm{R}} 29.8 \mathrm{~min}(52.1 \%)$ (Major diastereomer)

$33 t_{\mathrm{R}} 26.7 \mathrm{~min}(8.6 \%) ; t_{\mathrm{R}} 30.1 \mathrm{~min}$ (91.4\%) (Diacel Chiralpak AS-H 0.46 cm $\phi$ x 25 cm, Gradient; $t_{0} 0 \%$ IPA in Hexane, $t_{10 \min } 2 \%$ IPA in Hexane, $t_{20 \min } 4 \%$ IPA in Hexane, $t_{30 \min } 6 \%$ IPA in Hexane, $1 \mathrm{~mL} / \mathrm{min}$ ) 

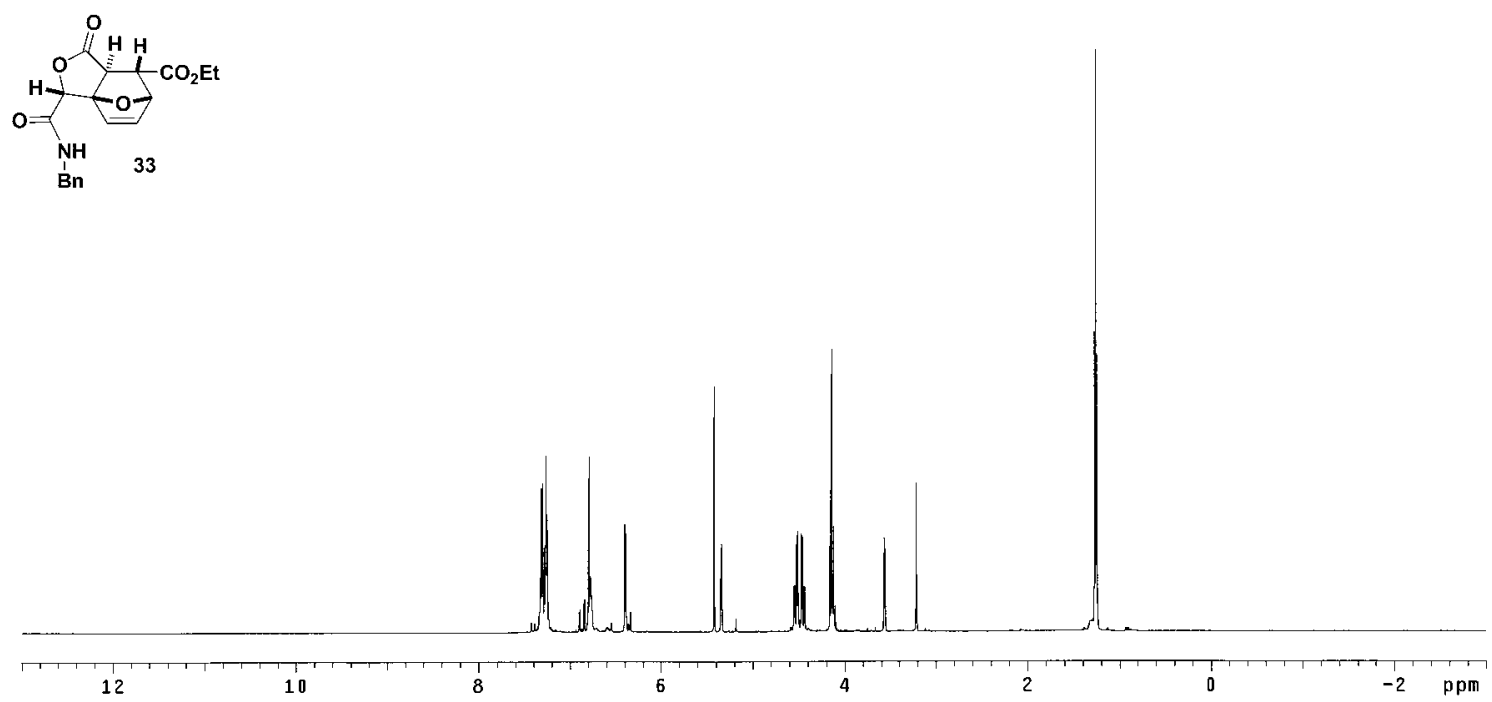

\section{Synthesis of (tert-butylcarbamoyl)(5-bromofuran-2-yl)methyl benzoate (x-ray)}

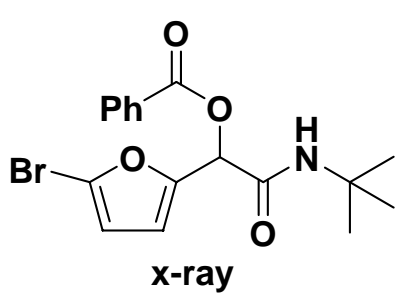

Following General Procedure II, a mixture of tertbutyisocyanide (13) (9.5 mg, $13 \mu \mathrm{L}, 0.11 \mathrm{mmol}, 1.0$ equiv), benzoic acid (5) (14.6 mg, $0.12 \mathrm{mmol}, 1.05$ equiv) and 5bromo-2-furaldehyde (21 mg, $0.12 \mathrm{mmol}, 1.05$ equiv) in

$\mathrm{CH}_{2} \mathrm{Cl}_{2}$ was stirred for 12 hrs. After removal of solvent and silica gel chromatography, 25 (143 mg, 97\%) was obtained as a whitish-brown solid. The compound was crystallized from EtOAc:Hex; 1:4.

Data:

${ }^{1}$ H NMR: $\quad\left(400 \mathrm{MHz}, \mathrm{CDCl}_{3}\right)$

$\delta$ 8.08-8.06 (dd, $J=0.4,8.0 \mathrm{~Hz}, 2 \mathrm{H}$, aryl); 7.63-7.60 (t, $J=7.2 \mathrm{~Hz}, 1 \mathrm{H}$, aryl); 7.50-7.46 (t, $J=8.0 \mathrm{~Hz}, 2 \mathrm{H}$, aryl); $6.55(\mathrm{~d}, J=4.0 \mathrm{~Hz}, 1 \mathrm{H}$, aryl); 6.31 (d, $J=3.2 \mathrm{~Hz}, 1 \mathrm{H}$, aryl); 6.26 (s, 1H, CH); 6.08 (bs, 1H, NH); 1.40 (s, $\left.9 \mathrm{H}, \mathrm{CH}_{3}\right)$ 
${ }^{13}$ C NMR: $\quad\left(100 \mathrm{MHz}, \mathrm{CDCl}_{3}\right)$

$\delta 164.9,164.7,150.6,134.0,130.1,129.1,128.9,123.5,114.4,112.7$,

69.2, 52.1, 28.9

TLC: $\quad \mathrm{R} f=0.53($ EtOAc:Hex 1:2)

HRMS: $\quad$ EIMS calculated for $\mathrm{C}_{17} \mathrm{H}_{18} \mathrm{BrNO}_{4}{ }^{+}[\mathrm{M}+\mathrm{H}]^{+}: 380.0497$, found: 380.0488

HPLC: $\quad t_{\mathrm{R}} 5.6 \mathrm{~min}(49.2 \%) ; t_{\mathrm{R}} 6.5 \mathrm{~min}(50.8 \%)$

$t_{\mathrm{R}} 5.7 \min (1.8 \%) ; t_{\mathrm{R}} 6.8 \min$ (98.2\%) (Diacel Chiralpak AS-H $0.46 \mathrm{~cm} \phi \mathrm{x}$

$25 \mathrm{~cm}, t_{0 \min } 5 \%$ IPA in Hexane, $t_{5 \min } 7 \%$ IPA in Hexane, $t_{10 \min } 9 \%$ IPA in

Hexane, $t_{13 \min } 11 \%$ IPA in Hexane, $1 \mathrm{~mL} / \mathrm{min}$ )

\section{$\underline{\text { X-Ray }}$}

Data:
Identification code

Empirical formula

Formula weight

Temperature

Wavelength

Crystal system

Space group

Unit cell dimensions

Volume

Z

Density (calculated)

Absorption coefficient

$\mathrm{F}(000)$

Crystal size

Theta range for data collection

Index ranges

Reflections collected

Independent reflections

Completeness to theta $=25.00^{\circ}$ sls82t

C17 H18 Br N O4

380.23

193(2) K

$0.71073 \AA$

Orthorhombic

P2(1)2(1)2(1)

$\mathrm{a}=9.4372(8) \AA \alpha=90^{\circ}$.

$\mathrm{b}=20.8127(16) \AA \beta=90^{\circ}$.

$\mathrm{c}=36.610(3) \AA \gamma=90^{\circ}$.

7190.7(10) $\AA^{3}$

16

$1.405 \mathrm{Mg} / \mathrm{m}^{3}$

$2.304 \mathrm{~mm}^{-1}$

3104

$0.08 \times 0.08 \times 0.04 \mathrm{~mm}^{3}$

1.93 to $25.00^{\circ}$.

$-11<=\mathrm{h}<=9,-18<=\mathrm{k}<=24$,

$-43<=\mathrm{l}<=43$

41295

12659 [R(int) $=0.1129]$

$100.0 \%$ 
Absorption correction

Max. and min. transmission

Refinement method

Data / restraints / parameters

Goodness-of-fit on $\mathrm{F}^{2}$

Final $\mathrm{R}$ indices [I $>2 \operatorname{sigma}(\mathrm{I})]$

$\mathrm{R}$ indices (all data)

Absolute structure parameter

Largest diff. peak and hole
Empirical

0.9135 and 0.8371

Full-matrix least-squares on $\mathrm{F}^{2}$

12659 / 0 / 829

0.986

$\mathrm{R} 1=0.0622, \mathrm{wR} 2=0.0992$

$\mathrm{R} 1=0.1420, \mathrm{wR} 2=0.1229$

$-0.015(9)$

0.930 and -0.748 e. $\AA^{-3}$

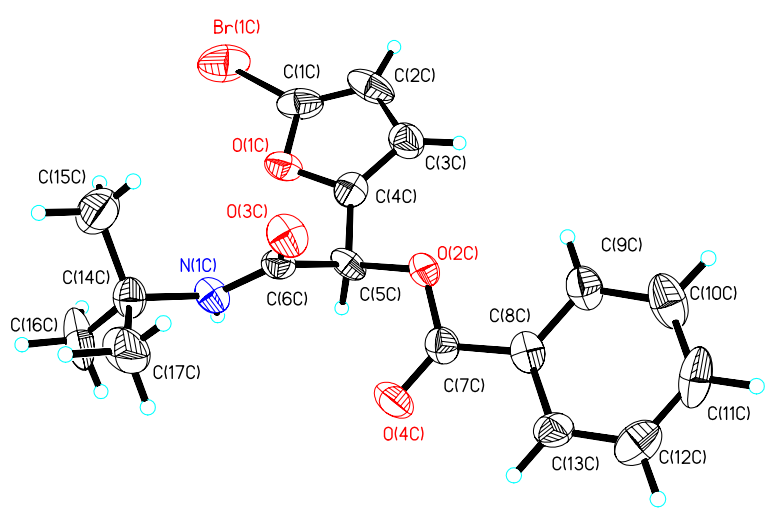

